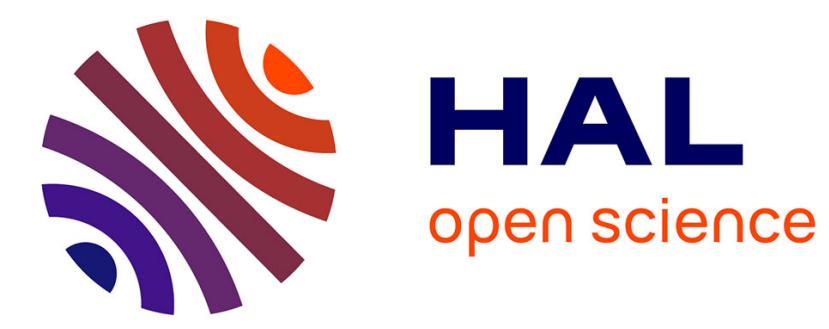

\title{
Multivariate reciprocal inverse Gaussian distributions from the Sabot-Tarrès-Zeng integral
}

Gérard Letac, Jacek Wesolowski

\section{To cite this version:}

Gérard Letac, Jacek Wesolowski. Multivariate reciprocal inverse Gaussian distributions from the Sabot-Tarrès-Zeng integral. Journal of Multivariate Analysis, 2020, 175, pp.104559 10.1016/j.jmva.2019.104559 . hal-03488550

\section{HAL Id: hal-03488550 \\ https://hal.science/hal-03488550}

Submitted on 21 Dec 2021

HAL is a multi-disciplinary open access archive for the deposit and dissemination of scientific research documents, whether they are published or not. The documents may come from teaching and research institutions in France or abroad, or from public or private research centers.
L'archive ouverte pluridisciplinaire HAL, est destinée au dépôt et à la diffusion de documents scientifiques de niveau recherche, publiés ou non, émanant des établissements d'enseignement et de recherche français ou étrangers, des laboratoires publics ou privés.

\section{다)(1) $(5$}

Distributed under a Creative Commons Attribution - NonCommerciall 4.0 International 
AMS CLASSIFICATION: Primary 60E05, secondary 62E10.

AbBREviated TITLE: $M R I G_{n}$ distributions

\title{
Multivariate reciprocal inverse Gaussian distributions from the Sabot -Tarrès -Zeng integral
}

\author{
Gérard Letac $^{\mathrm{a}}$, Jacek Wesołowski ${ }^{\mathrm{b}}$
}

${ }^{a}$ Institut de Mathématiques de Toulouse, Université Paul Sabatier, 31062, Toulouse, France.

${ }^{b}$ Matematyki i Nauk Informacyjnych, Politechnika Warszawska, Warszawa, Poland.

\section{Abstract}

In Sabot and Tarrès [14], the authors have explicitly computed the integral

$$
S T Z_{n}=\int \exp (-\langle x, y\rangle)\left(\operatorname{det} M_{x}\right)^{-1 / 2} d x
$$

where $M_{x}$ is a symmetric matrix of order $n$ with fixed non-positive off-diagonal coefficients and with diagonal $\left(2 x_{1}, \ldots, 2 x_{n}\right)$. The domain of integration is the part of $\mathbb{R}^{n}$ for which $M_{x}$ is positive definite. We calculate more generally for $b_{1} \geq 0, \ldots b_{n} \geq 0$ the integral

$$
\int \exp \left(-\langle x, y\rangle-\frac{1}{2} b^{\top} M_{x}^{-1} b\right)\left(\operatorname{det} M_{x}\right)^{-1 / 2} d x,
$$

we show that it leads to a natural family of distributions in $\mathbb{R}^{n}$, called the $M R I G_{n}$ probability laws. This family is stable by marginalization and by conditioning, and it has number of properties which are multivariate versions of familiar properties of univariate reciprocal inverse Gaussian distribution. In general, if the power of $\operatorname{det} M_{x}$ under the integral in $S T Z_{n}$ is distinct from $-1 / 2$ it is not known how to compute the integral. However, introducing the graph $G$ having $V=\{1, \ldots, n\}$ for set of vertices and the set $E$ of $\{i, j\}^{\prime}$ s of non-zero entries of $M_{x}$ as set of edges, we show also that in the particular case where $G$ is a tree, the integral

$$
\int \exp (-\langle x, y\rangle)\left(\operatorname{det} M_{x}\right)^{q-1} d x
$$

where $q>0$, is computable in terms of the MacDonald function $K_{q}$.

Keywords: Laplacian of a graph, MacDonald function, multivariate reciprocal inverse Gaussian, supersymmetry. AMS classification: primary 60E05, secondary 62 E10.

\section{Introduction: the Sabot -Tarrès -Zeng integral.}

Let us describe the integral appearing in Sabot and Tarrès [14]. Let $W=\left(w_{i j}\right)_{1 \leq i, j \leq n}$ be a symmetric matrix such that $w_{i i}=0$ for all $i=1, \ldots, n$ and such that $w_{i j} \geq 0$ for $i \neq j$. For $x=\left(x_{1}, \ldots, x_{n}\right) \in \mathbb{R}^{n}$ define the matrix $M_{x}=2 \operatorname{diag}\left(x_{1}, \ldots, x_{n}\right)-W$. For instance if $n=3$ we have

$$
M_{x}=\left[\begin{array}{ccc}
2 x_{1} & -w_{12} & -w_{13} \\
-w_{12} & 2 x_{2} & -w_{23} \\
-w_{13} & -w_{23} & 2 x_{3}
\end{array}\right]
$$

Email addresses: gerard.letac@math.univ-toulouse.fr (Gérard Letac), wesolo@mini.pw.edu.pl (Jacek Wesołowski) 
Denote by $C_{W}$ the set of $x \in \mathbb{R}^{n}$ such that $M_{x}$ is positive definite. It is easy to see that $C_{W}$ is an open non-empty unbounded convex set. This is not a cone in general. Frequently we consider the undirected graph $G$ with set of vertices $\{1, \ldots, n\}$ and with set of edges $E=\left\{\{i, j\} ; w_{i j}>0\right\}$ and we speak of the graph $G$ associated to $W$. The Sabot-Tarrès-Zeng integral is, for $y_{1}, \ldots, y_{n}>0$

$$
S T Z_{n}=\int_{C_{W}} e^{-\left(x_{1} y_{1}+\cdots+x_{n} y_{n}\right)} \frac{d x_{1} \times \cdots \times d x_{n}}{\sqrt{\operatorname{det} M_{x}}}=\left(\sqrt{\frac{\pi}{2}}\right)^{n} \frac{1}{\sqrt{y_{1} \times \cdots \times y_{n}}} e^{-\frac{1}{2} \sum_{i j} w_{i j} \sqrt{y_{i} y_{j}}} .
$$

Sabot and Tarrès [14] give a probabilistic proof of this remarkable result. Another proof is in Sabot, Tarrès and Zeng [17], based on the Cholesky decomposition. This integral leads naturally to consideration of probability laws on $\mathbb{R}^{n}$ that we call $S T Z_{n}$ distributions with densities proportional to $e^{-\langle x, y\rangle}\left(\operatorname{det} M_{x}\right)^{-1 / 2} 1_{C_{W}}(x)$. In the present paper we derive, using a different approach than the two methods mentioned above, a more general $M R I G_{n}$ integral in Theorem 2.2. In particular, we give a new proof of (1). The symbol $M R I G$ for multivariate reciprocal inverse Gaussian, is explained below.

This $M R I G_{n}$ integral enables us to create a new set (called the $M R I G_{n}$ family) of distributions on $\mathbb{R}^{n}$ which is stable by marginalization and, up to a translation, stable by conditioning. The bibliography concerning the appearance of the $S T Z_{n}$ and $M R I G_{n}$ laws in probability theory is already very rich and we suggest to look at Sabot and Zeng [15] and Disertori, Merkl and Rolles [8] for many references. An unpublished observation of 2015 of the first author has been used and reproved in these two publications and some facts of the present paper can be found in them. However, we use here only elementary methods to get our results.

Let us recall that in literature, the generalized inverse Gaussian distributions $\operatorname{GIG}(a, b, q)$ are one dimensional laws with density proportional to $e^{-a^{2} x-\frac{b^{2}}{4 x}} x^{q-1} 1_{(0, \infty)}(x)$, for $a, b>0$ and $q$ real (see Seshadri [18] for instance). Parameterizations differ according to the needs of authors and we have chosen an appropriate one in the present paper. The most famous particular case is for $q=-1 / 2$ with the inverse Gaussian distribution. A random variable $Y$ with the inverse Gaussian distribution $I G(a, b)=G I G(a / 2,2 b,-1 / 2)$ has Laplace transform

$$
\mathbb{E}\left(e^{-s Y}\right)=e^{b\left(a-\sqrt{a^{2}+s}\right)}
$$

for $s>-a^{2}$. Its density is proportional to $e^{-\frac{a^{2} y}{4}-\frac{b^{2}}{y}} y^{-3 / 2} 1_{(0, \infty)}(y)$. A less known case, but the important one for the present paper, is for $q=1 / 2$ with the reciprocal inverse Gaussian distribution. Actually, it is a distribution of the inverse of a random variable with an $I G$ distribution. A random variable $X$ with a reciprocal inverse Gaussian distribution $\operatorname{RIG}(a, b)=G I G(a, b, 1 / 2)$ has Laplace transform for $s>-a^{2}$

$$
\mathbb{E}\left(e^{-s X}\right)=\frac{a}{\sqrt{a^{2}+s}} e^{b\left(a-\sqrt{a^{2}+s}\right)}
$$

and is such that

$$
\mathbb{E}(X)=m=\frac{a b+1}{2 a^{2}}, \mathbb{E}\left(X^{2}\right)=\frac{1}{4 a^{4}}\left(a^{2} b^{2}+3 a b+3\right), \operatorname{Var}(X)=\frac{a b+2}{4 a^{4}} .
$$

Its density is proportional to $e^{-a^{2} x-\frac{b^{2}}{4 x}} x^{-1 / 2} 1_{(0, \infty)}(x)$. This law is considered for instance in Barndorff-Nielsen and Koudou [3]. Our $M R I G_{n}$ distributions have some properties which are multivariate versions of properties known for the univariate $R I G$ law. These are good reasons for attaching the name multivariate ( $n$-dimensional) $R I G$ to the members of this family. A particular case of the family $M R I G_{2}$ appears in Barndorff-Nielsen, Blaesild and Seshadri [2]. The family $S T Z_{2}$ appears in Barndorff-Nielsen and Rysberg [4].

Section 2 proves and comments on the $M R I G_{n}$ integral, including a presentation of the DisertoriSpencer-Zinbauer [7] and Disertori-Merkles-Rolles [8] integrals in the studies of supersymmetry. Section 3 gives some examples. Section 4 details the properties of the $M R I G_{n}$ laws (we carefully distinguish along the paper the $M R I G_{n}$ integral and the $M R I G_{n}$ laws). Section 5 considers the particular case of the $S T Z_{n}$ integral when the graph $G$ associated to $W$ is a tree. Then we generalize the $S T Z_{n}$ integral by computing in this case $\int_{C_{W}} \exp (-\langle x, y\rangle)\left(\operatorname{det} M_{x}\right)^{q-1} d x$ and thus, in particular, obtaining the norming constant for the density considered in Massam and Wesołowski [11]. Interestingly enough, this generalization allows us to 
not restrict to the case where the $w_{i j}$ 's are non-negative. The reason is the not so well known fact: if the associated graph of a positive definite matrix $M=\left(m_{i j}\right)$ is a tree then the symmetric matrix $M^{\prime}=\left( \pm m_{i j}\right)$ is still positive definite whatever the \pm are outside of the diagonal; therefore $C_{W}$ is unchanged. Section 6 mentions a striking consequence (Corollary 6.2) of the $M R I G_{n}$ integral: if $\left(B_{1}, \ldots, B_{n}\right)$ is multivariate normal, i.e. $\left(B_{1}, \ldots, B_{n}\right) \sim N\left(0, M_{x}\right)$, then

$$
\operatorname{Pr}\left(B_{1}>0, \ldots, B_{n}>0\right)=\frac{1}{(2 \pi)^{n / 2}} \int_{C_{W} \cap\{t \leq x\}} \frac{d t}{\sqrt{\left(x_{1}-t_{1}\right) \cdots\left(x_{n}-t_{n}\right)} \sqrt{\operatorname{det} M_{t}}} .
$$

Section 7 proves a marginal but delicate fact that the densities of the $M R I G_{n}$ distributions are continuous on the whole $\mathbb{R}^{n}$. A first version of this paper is on arXiv 1709.04843.

\section{The $M R I G_{n}$ integral}

\subsection{The integral and its various forms}

It is useful to recall a classical formula, which is in fact the particular case $n=1$ of Theorem 2.2 below and the starting point of an induction proof.

Lemma 2.1. If $a>0$ and $b \geq 0$ then

$$
\int_{0}^{\infty} e^{-\frac{a^{2} t^{2}}{2}-\frac{b^{2}}{2 t^{2}}} d t=\sqrt{\frac{\pi}{2}} \frac{1}{a} e^{-a b}
$$

Various proofs of Lemma 2.1 exist in the literature. An elegant one considers the equivalent formulation

$$
\frac{a}{\sqrt{2 \pi}} \int_{-\infty}^{\infty} \exp \left[-\frac{1}{2}\left(a t-\frac{b}{t}\right)^{2}\right] d t=1
$$

and proves (6) by the change of variable $x=\varphi(t)=t-\frac{b}{a t}$ which preserves the Lebesgue measure on $\mathbb{R}$. This idea seems to be due to George Boole [6]. In the sequel, if $a$ is a column vector, or more generally a matrix, then $a^{\top}$ denotes the transposed matrix of $a$.

Theorem 2.2. Let $a_{1}, \ldots, a_{n}>0$ and $b_{1}, \ldots, b_{n} \geq 0$. Then with $a=\left(a_{1}, \ldots, a_{n}\right)^{\top}$ and $b=\left(b_{1}, \ldots, b_{n}\right)^{\top}$ we have

$$
M R I G_{n}=\int_{C_{W}} e^{-\frac{1}{2}\left(a^{\top} M_{x} a+b^{\top} M_{x}^{-1} b\right)} \frac{d x}{\sqrt{\operatorname{det} M_{x}}}=\left(\frac{\pi}{2}\right)^{n / 2} \frac{e^{-\left(a_{1} b_{1}+\cdots+a_{n} b_{n}\right)}}{a_{1} \times \cdots \times a_{n}}
$$

\section{Comments.}

- Inserting $t=\sqrt{2 x}$ in (5) we see that (5) is the particular case $n=1$ of (7).

- Remarkably, the right hand side of (7) does not depend on $W$.

- Another presentation of (7) is

$$
\int_{C_{W}} \exp \left(-\frac{1}{2}\left\|M_{x}^{1 / 2} a-M_{x}^{-1 / 2} b\right\|^{2}\right) \frac{d x}{\sqrt{\operatorname{det} M_{x}}}=\left(\frac{\pi}{2}\right)^{n / 2} \frac{1}{a_{1} \times \cdots \times a_{n}} .
$$

For $n=1$ this is nothing but (6) after the change of variable $x=t^{2} / 2$.

- Another variation: from (23) below, writing for short $\sqrt{s}=\left(\sqrt{s_{1}}, \ldots, \sqrt{s_{n}}\right)^{\top}$ we have

$$
\left(\frac{2}{\pi}\right)^{n / 2} \int_{C_{W}} e^{-\langle x, s\rangle-\frac{1}{2} b^{\top} M_{x}^{-1} b} \frac{d x}{\sqrt{\operatorname{det} M_{x}}}=\frac{1}{\sqrt{s_{1} \times \cdots \times s_{n}}} e^{-2\langle b, \sqrt{s}\rangle-\frac{1}{2} \sqrt{s} W \sqrt{s}} .
$$


- One more variation of (7) and (23) is obtained by considering a positive definite matrix $A=\left(a_{i j}\right)_{1 \leq i, j \leq n}$ in the formula

$$
\left(\frac{2}{\pi}\right)^{n / 2} \int_{C_{W}} e^{-\frac{1}{2} \operatorname{tr}\left(M_{x} A\right)-\frac{1}{2} b^{\top} M_{x}^{-1} b} \frac{d x}{\sqrt{\operatorname{det} M_{x}}}=\frac{1}{\sqrt{a_{11} \times \cdots \times a_{n n}}} e^{-\left(b_{1} \sqrt{a_{11}}+\cdots+b_{n} \sqrt{a_{n n}}\right)-\frac{1}{2} \sum_{i, j=1}^{n} w_{i j}\left(\sqrt{a_{i i} a_{j j}}-a_{i j}\right)} .
$$

If $A=\Sigma^{-1}$, consider the Gaussian random variable $X=\left(X_{1}, \ldots, X_{n}\right) \sim N(0, \Sigma)$. Recall that $\rho_{i j}=$ $-a_{i j} / \sqrt{a_{i i} a_{j j}}$ is the correlation between $X_{i}$ and $X_{j}$ conditioned by all $\left(X_{k} ; k \neq i, j\right)$. Therefore

$$
\sum_{i, j=1}^{n} w_{i j}\left(\sqrt{a_{i i} a_{j j}}-a_{i j}\right)=\sum_{i, j=1}^{n} w_{i j} \sqrt{a_{i i} a_{j j}}\left(1+\rho_{i j}\right)
$$

- In (23) the condition $a_{1}, \ldots a_{n}>0$ is easily relaxed to $a_{1}, \ldots, a_{n} \neq 0$ : in the right hand side of $a_{1}, \ldots a_{n}>0$ replace $a_{i}$ by $\left|a_{i}\right|$, Things are quite different for the condition $b_{1}, \ldots b_{n} \geq 0$ : see the comments of the example $n=2$ in Section 3.

\subsection{Proof of Theorem 2.2}

Proof. We prove it by induction on $n$. As mentioned above, Lemma 2.1 is the case $n=1$. Assume that the result is true for $n$. Consider

$$
W^{1}=\left[\begin{array}{cc}
W & c \\
c^{\top} & 0
\end{array}\right], M^{1}=\left[\begin{array}{cc}
M_{x} & -c \\
-c^{\top} & 2 x_{n+1}
\end{array}\right]
$$

where $c=\left(c_{1}, \ldots, c_{n}\right)^{\top}$ with $c_{i} \geq 0$ for all $i$. We now assume that $\left(x, x_{n+1}\right) \in C_{W^{1}}$. From the positive definiteness of $M^{1}$ we see that the Schur complement $t^{2}=2 x_{n+1}-c^{\top} M_{x}^{-1} c$ is positive. We write

$$
M^{1}=\left[\begin{array}{cc}
I_{n} & 0 \\
-c^{\top} M_{x}^{-1} & 1
\end{array}\right]\left[\begin{array}{cc}
M_{x} & 0 \\
0 & t^{2}
\end{array}\right]\left[\begin{array}{cc}
I_{n} & -M_{x}^{-1} c \\
0 & 1
\end{array}\right]
$$

Equality (9) leads to the computation of $\left(M^{1}\right)^{-1}$ as follows:

$$
\left(M^{1}\right)^{-1}=\left[\begin{array}{cc}
I_{n} & M_{x}^{-1} c \\
0 & 1
\end{array}\right]\left[\begin{array}{cc}
M_{x}^{-1} & 0 \\
0 & t^{-2}
\end{array}\right]\left[\begin{array}{cc}
I_{n} & 0 \\
c^{\top} M_{x}^{-1} & 1
\end{array}\right]=\left[\begin{array}{cc}
M_{x}^{-1}+t^{-2} M_{x}^{-1} c c^{\top} M_{x}^{-1} & t^{-2} M_{x}^{-1} c \\
t^{-2} c^{\top} M_{x}^{-1} & t^{-2}
\end{array}\right] .
$$

Before writing down the integral $M R I G_{n+1}$ we observe that

$$
\begin{aligned}
\left(a^{\top}, a_{n+1}\right) M^{1}\left(\begin{array}{c}
a \\
a_{n+1}
\end{array}\right) & =a^{\top} M_{x} a-2 a^{\top} c a_{n+1}+2 x_{n+1} a_{n+1}^{2} \\
& =-2 a^{\top} c a_{n+1}+a^{\top} M_{x} a+c^{\top} M_{x}^{-1} c a_{n+1}^{2}+t^{2} a_{n+1}^{2} \\
\left(b^{\top}, b_{n+1}\right)\left(M^{1}\right)^{-1}\left(\begin{array}{c}
b \\
b_{n+1}
\end{array}\right)= & b^{\top} M_{x}^{-1} b+t^{-2} b^{\top} M_{x}^{-1} c c^{\top} M_{x}^{-1} b+2 t^{-2} b^{\top} M_{x}^{-1} c b_{n+1}+t^{-2} b_{n+1}^{2} \\
= & b^{\top} M_{x}^{-1} b+t^{-2}\left(b_{n+1}+b^{\top} M_{x}^{-1} c\right)^{2} .
\end{aligned}
$$

Also observe that the convex set $C_{W^{1}}$ is parameterized by $(x, t)$ in $C_{W} \times(0, \infty)$ and that, from (9) we have $\operatorname{det} M^{1}=t^{2} \operatorname{det} M_{x}$. With this parameterization we have

$$
\frac{d x d x_{n+1}}{\sqrt{\operatorname{det} M^{1}}}=\frac{d x}{\sqrt{\operatorname{det} M_{x}}} d t
$$

We now write $M R I G_{n+1}$ as follows 


$$
\begin{aligned}
& M R I G_{n+1}=e^{a^{\top} c a_{n+1}} \int_{C_{W}} \exp -\frac{1}{2}\left[a^{\top} M_{x} a+c^{\top} M_{x}^{-1} c a_{n+1}^{2}+b^{\top} M_{x}^{-1} b\right] \\
& \times\left(\int_{0}^{\infty} \exp -\frac{1}{2}\left[t^{2} a_{n+1}^{2}+t^{-2}\left(b_{n+1}+b^{\top} M_{x}^{-1} c\right)^{2}\right] d t\right) \frac{d x}{\sqrt{\operatorname{det} M_{x}}} \\
& =\sqrt{\frac{\pi}{2}} \frac{1}{a_{n+1}} e^{a^{\top} c a_{n+1}-a_{n+1} b_{n+1}} \int_{C_{W}} \exp -\frac{1}{2}\left[a^{\top} M_{x} a+\left(c^{\top} a_{n+1}+b^{\top}\right) M_{x}^{-1}\left(c a_{n+1}+b\right)\right] \frac{d x}{\sqrt{\operatorname{det} M_{x}}} \\
& =\left(\frac{\pi}{2}\right)^{(n+1) / 2} \frac{1}{a_{1} \times \cdots \times a_{n+1}} e^{-a^{\top} b-a_{n+1} b_{n+1}} \text {. }
\end{aligned}
$$

In this chain of equalities (11) is a consequence of Lemma 2.1 applied to the pair

$$
a_{n+1}, b_{n+1}+b^{\top} M_{x}^{-1} c .
$$

Here a comment is in order: a famous lemma of Stieltjes implies that $M_{x}^{-1}$ has non-negative coefficients when $x \in C_{W}$. Let us detail the proof in this particular case: if $D=2 \operatorname{diag}\left(x_{1}, \ldots, x_{n}\right)$ then $M_{x}=D^{1 / 2}\left(I_{n}-\right.$ A) $D^{1 / 2}$ where $A=D^{-1 / 2} W D^{-1 / 2}$. Since $M_{x}$ is positive definite, $I_{n}-A$ is also positive definite. Now write $\left(I_{n}-A\right)^{-1}=I_{n}+A+\cdots+A^{2 N-1}+A^{N}\left(I_{n}-A\right)^{-1} A^{N}$. Since $A^{N}\left(I_{n}-A\right)^{-1} A^{N}$ is positive semidefinite, its trace is $\geq 0$ and therefore for all $N$

$$
\sum_{k=0}^{2 N-1} \operatorname{tr}\left(A^{k}\right) \leq \operatorname{tr}\left(I_{n}-A\right)^{-1}
$$

Since $A$ has non-negative coefficients this implies that $\sum_{k=0}^{\infty} \operatorname{tr}\left(A^{k}\right)$ converges. In particular $\lim _{N \rightarrow \infty} \operatorname{tr}\left(A^{2 N}\right)=$ 0 . This implies that all the eigenvalues of $A$ are in $(-1,1)$ and therefore the series of matrices $S=\sum_{k=0}^{\infty} A^{k}$ converges to $\left(I_{n}-A\right)^{-1}$. Since $A$ has non-negative coefficients the same is true for $S$ and for $M_{x}^{-1}=$ $D^{-1 / 2} S D^{-1 / 2}$. Furthermore, if the graph $G$ has vertices $\{1, \ldots, n\}$ and has edges $\{i, j\}$ present according to the fact that $a_{i j}>0$ or not, then $\left(I_{n}-A\right)^{-1}$ is positive definite if $G$ is connected (this remark will be used in the proof of Lemma 2.4 below).

As a consequence $b_{n+1}+b^{\top} M_{x}^{-1} c \geq 0$ and therefore (5) is applicable. Equality (12) is a consequence of the induction hypothesis where the pair $(a, b)$ is replaced by $\left(a, a_{n+1} c+b\right)$. The induction hypothesis is extended.

\subsection{Laplacian of $W$ and parameterizations of $C_{W}$ by $(0, \infty)^{n}$ and $\mathbb{R}^{n}$}

In order to show in Section 2.4 that two other remarkable integrals can be deduced from the $M R I G_{n}$ integral (7), it is necessary to recall some definitions about Laplacian on graphs or weighted graphs (see for instance Bapat [1]). We define the Laplacian of $W$ as the quadratic form on $\mathbb{R}^{n}$ defined by

$$
v^{\top} L_{W} v=\sum_{i<j} w_{i j}\left(v_{i}-v_{j}\right)^{2}=\frac{1}{2} \sum_{i=1}^{n} \sum_{j=1}^{n} w_{i j}\left(v_{i}-v_{j}\right)^{2} .
$$

If $s_{i}=\sum_{j=1}^{n} w_{i j}$ and if $D=\operatorname{diag}\left(s_{1}, \ldots, s_{n}\right)$ the representative matrix of this quadratic form is $L_{W}=D-W$. From the definition it is semi positive definite, and since $(1, \ldots, 1)^{\top}$ is a eigenvector of $L_{W}$ associated to the the eigenvalue zero, $L_{W}$ cannot be positive definite. However, by adding a proper diagonal matrix

$$
D_{b}=\operatorname{diag}\left(b_{1}, \ldots, b_{n}\right)
$$

with $b_{i} \geq 0$ the matrix $D_{b}+L_{W}$ can be positive definite. One can also remark that $W^{1}=\left[\begin{array}{cc}W & b \\ b^{\top} & 0\end{array}\right]$ implies that $L_{W^{1}}=\left[\begin{array}{cc}D_{b}+L_{W} & -b \\ -b^{\top} & \sum_{j=1}^{n} b_{j}\end{array}\right]$.

Lemma 2.3. $D_{b}+L_{W}$ is positive definite if and only if for each connected component $C$ of the graph associated to $W$ there exists $k \in C$ such that $b_{k}>0$. 
Proof. $\Leftarrow$ Enough is to assume that the associated graph is connected and that there exists a $k$ such that $b_{k}>0$. If $v$ is such that $v^{\top}\left(D_{b}+L_{W}\right) v=0$ then $v_{k}=0$. Furthermore $v_{i}-v_{j}=0$ if $w_{i j}>0$. Since the associated graph is connected all the $v_{i}$ 's are equal, and they are zero like $v_{k}:$ this shows the positive definiteness of $D_{b}+L_{W} . \Rightarrow$ Here again we can assume that $G$ is connected. We have seen that if $b_{i}=0$ for all $i$ then $D_{b}+L_{W}=L_{W}$ cannot be positive definite.

The next lemma describes an important parameterization of $C_{W}$ by $(0, \infty)^{n}$. Note that it depends on a non-zero parameter $b=\left(b_{1}, \ldots, b_{n}\right)^{\top} \in[0, \infty)^{n}$. The case $b=(1, \ldots, 1)^{\top}$ is most useful.

Lemma 2.4. Assume that the graph $G$ associated to $W$ is connected. Let $y \in(0, \infty)^{n}$, fix $b \in[0, \infty)^{n}$ such that $b \neq 0$ and define $x \in \mathbb{R}^{n}$ by

$$
2 x_{i}=\frac{1}{y_{i}}\left(b_{i}+\sum_{j=1}^{n} w_{i j} y_{j}\right)
$$

Then $x$ belongs to $C_{W}$, we have $M_{x}=D_{b} D_{y}^{-1}+L_{W}$ and $y=M_{x}^{-1} b$, the map $y \mapsto x$ is a diffeomorphism from $(0, \infty)^{n}$ onto $C_{W}$ and

$$
d x=\frac{\operatorname{det} M_{x}}{2^{n}} \frac{d y}{y_{1} \times \cdots \times y_{n}} .
$$

Proof. We rewrite (14) as $2 x_{i} y_{i}-\sum_{j=1}^{n} w_{i j} y_{j}=b_{i}$ and thus it is equivalent to $b=M_{x} y$. Denote

$$
W^{(y)}=D_{y} W D_{y}
$$

and $s_{i}^{(y)}=\sum_{j=1}^{n} w_{i j} y_{i} y_{j}=2 x_{i} y_{i}^{2}-b_{i} y_{i}$. Therefore

$$
D_{s^{(y)}}=2 D_{y} D_{x} D_{y}-D_{b} D_{y}, \quad L_{W^{(y)}}=D_{s^{(v)}}-W^{(y)}=2 D_{y} D_{x} D_{y}-D_{b} D_{y}-D_{y} W D_{y} .
$$

From the definition (13) of the Laplacian we have $L_{W^{(y)}}=D_{y} L_{W} D_{y}$ and we get

$$
D_{b} D_{y}+L_{W^{(y)}}=2 D_{y} D_{x} D_{y}-D_{y} W D_{y}, \quad D_{b} D_{y}^{-1}+L_{W}=M_{x} .
$$

From Lemma 2.3 $M_{x}=D_{b} D_{y}^{-1}+L_{W}$ is positive definite and furthermore $y=M_{x}^{-1} b$. Equality $b=M_{x} y$ shows that the map $y \mapsto x$ from $(0, \infty)^{n}$ to $C_{W}$ is injective since $0=M_{x}\left(y-y^{\prime}\right)$ implies $y=y^{\prime}$ from the definite positiveness of $M_{x}$. If $x \in C_{W}$, define $y=M_{x}^{-1} b$. The fact that $M_{x}^{-1}$ has only non-negative coefficients implies that $y \in[0, \infty)^{n}$. The fact that $G$ is connected implies that $y \in(0, \infty)^{n}$. We get $M_{x}=D_{b} D_{y}^{-1}+L_{W}$ and this shows the surjectivity since any $y \in(0, \infty)$ provides a positive definite matrix $D_{b} D_{y}^{-1}+L_{W}$. The fact that $y \mapsto x$ is a diffeomorphism from $(0, \infty)^{n}$ onto $C_{W}$ is clear.

The differential of the map $y \mapsto x$ from $C_{W}$ onto $(0, \infty)^{n}$ is

$$
h \mapsto-2 M_{x}^{-1} D_{h} M_{x}^{-1} b=-2 M_{x}^{-1} D_{h} y .
$$

For showing (17) we observe that the differential of $M \mapsto M^{-1}$ is $H \mapsto-M^{-1} H M^{-1}$ and that the differential of the map $x \mapsto M_{x}$ is $h \mapsto 2 D_{h}$. The Jacobian of $y \mapsto x$ is therefore $\frac{2^{n}}{\operatorname{det} M_{x}} y_{1} \times \cdots \times y_{n}$ and this proves (15).

Replacing $y_{i}$ by $e^{t_{i}}$ we will use Lemma 2.4 in the next section under the following form:

Corollary 2.5. Under the hypothesis of Lemma 2.4 , for $t \in \mathbb{R}^{n}$ define

$$
2 x_{i}(t)=b_{i} e^{-t_{i}}+\sum_{j=1}^{n} w_{i j} e^{t_{j}-t_{i}} .
$$

Then the map $t \mapsto x=x(t)$ is a diffeomorphism from $\mathbb{R}^{n}$ onto $C_{W}$ and $d x=\frac{\operatorname{det} M_{x}}{2^{n}} d t$. 


\subsection{The Disertori-Spencer-Zirnbauer and Disertori-Merkl-Rolles integrals}

In application of Theorem 2.2 and Corollary 2.5, we prove two surprizing formulas $D S Z_{n}$ and $D M R_{n}$ due to Disertori, Spencer and Zirnbauer [7] and Disertori, Merkl and Rolles [8]. For describing them we need the following notations. We consider the quadratic form in (1.1) of the first paper:

$$
v^{\top} D(t) v=\sum_{1 \leq i<j \leq n} w_{i j} e^{t_{i}+t_{j}}\left(v_{i}-v_{j}\right)^{2}+\sum_{k=1}^{n} b_{k} e^{t_{k}} v_{k}^{2} .
$$

The element $(i, i)$ of the corresponding $n \times n$ matrix $D(t)$ is $b_{i} e^{t_{i}}+\sum_{j=1}^{n} w_{i j} e^{t_{i}+t_{j}}$ and the off diagonal element $(i, j)$ is $-w_{i j} e^{t_{i}+t_{j}}$. This is nothing but the quadratic form with matrix $D(t)=D_{b} D_{y}+L_{W^{(v)}}$ as in (16) when $y_{i}=e^{t_{i}}$ for all $i$.

We introduce a function $G(t)$ which is only marginally different from the $F$ defined by (1.2) in [7].

$$
G(t)=\sum_{i<j} w_{i j}\left(\cosh \left(t_{i}-t_{j}\right)-1\right)+\sum_{k=1}^{n}\left(\left(\cosh t_{k}-1\right) b_{k}+t_{k}\right) .
$$

With these notations, the surprising formula (1.4) of [7], see (19) below, is the subject of the following proposition.

Proposition 2.6. Assume that $W$ is such that the associated graph is connected and fix $b \in[0, \infty)^{n}$ with $b \neq 0$. Then

$$
D S Z_{n}=\frac{1}{(\sqrt{2 \pi})^{n}} \int_{\mathbb{R}^{n}} e^{-G(t)} \sqrt{\operatorname{det} D(t)} d t=1 .
$$

Proof. In (7) we insert $a_{1}=\ldots=a_{n}=1$ and we make the change of variable $x \mapsto t$ from $C_{W}$ onto $\mathbb{R}^{n}$ described in Corollary 2.5. We get

$$
\begin{aligned}
& -\frac{1}{2}\left(a^{\top} M_{x(t)} a+b^{\top} M_{x(t)}^{-1} b\right)=-\frac{1}{2} \sum_{i=1}^{n} 2 x_{i}(t)+\frac{1}{2} \sum_{i=1}^{n} \sum_{j=1}^{n} w_{i j}-\frac{1}{2} \sum_{i=1}^{n} b_{i} e^{t_{i}} \\
& =-\frac{1}{2} \sum_{i=1}^{n} b_{i}\left(e^{t_{i}}+e^{-t_{i}}\right)-\frac{1}{2} \sum_{i<j} w_{i j}\left(e^{t_{j}-t_{i}}+e^{t_{i}-t_{j}}-2\right)=-\sum_{i=1}^{n} b_{i} \cosh t_{i}-\sum_{i<j} w_{i j}\left(\cosh \left(t_{j}-t_{i}\right)-1\right) .
\end{aligned}
$$

Since $D(t)=D_{y(t)} M_{x(t)} D_{y(t)}$ we have $\operatorname{det} D(t)=e^{\sum_{i=1}^{n} 2 t_{i}} \operatorname{det} M_{x(t)}$. Using Corollary 2.5 we obtain (19).

Similarly, formula (2.4) of Disertori, Merkl and Rolles [8] introduces a probability $\mu(d s, d t)$ on $\mathbb{R}^{n} \times \mathbb{R}^{n}$ defined by

$$
\mu(d s, d t)=e^{-\frac{1}{2} s^{\top} D(t) s-G_{1}(t)} \operatorname{det} D(t) \frac{d t d s}{(2 \pi)^{n}}
$$

where the function $G_{1}$ is quite close to the function $G$ defined by (18) and is defined by

$$
G_{1}(t)=\sum_{i<j} w_{i j}\left(\cosh \left(t_{i}-t_{j}\right)-1\right)+\sum_{k=1}^{n}\left(e^{-t_{k}} b_{k}+t_{k}\right)=G(t)+\sum_{k=1}^{n}\left(1-\sinh t_{k}\right) b_{k} .
$$

If $(S, T) \sim \mu$ it is clear that $S$ is Gaussian when conditioned by $T$. However, the fact that the total mass of $\mu$ is one is not that obvious. The result is stated in Proposition 2.8 below. We skip its proof which uses again Corollary 2.5. It is a consequence of the $S T Z_{n}$ integral (1), by the change of variable of Corollary 2.5. The hypotheses on $W$ and $b$ are the same as in Proposition 2.6.

Proposition 2.7 Let $f(t)=\frac{1}{\left(\sqrt{2 \pi)^{n}}\right.} e^{-G_{1}(t)} \sqrt{\operatorname{det} D(t)}$. Then $f$ is a probability density on $\mathbb{R}^{n}$. Furthermore if $T \sim f(t) d t$ and $S \mid T \sim N\left(0, D(T)^{-1}\right)$ then $(S, T) \sim \mu$ defined by (20). 


\section{Examples}

The following examples consider various graphs associated to $W$ where some calculations about $M_{x}$ are explicit.

\subsection{The case $n=2$.}

We take

$$
W=\left[\begin{array}{ll}
0 & 1 \\
1 & 0
\end{array}\right], M_{x}=\left[\begin{array}{cc}
2 x_{1} & -1 \\
-1 & 2 x_{2}
\end{array}\right], M_{x}^{-1}=\frac{1}{4 x_{1} x_{2}-1}\left[\begin{array}{cc}
2 x_{2} & 1 \\
1 & 2 x_{1}
\end{array}\right]
$$

and $C_{W}$ is the convex set of $\mathbb{R}^{2}$ limited by one branch of a hyperbola

$$
C_{W}=\left\{\left(x_{1}, x_{2}\right) ; x_{1}, x_{2}>0,4 x_{1} x_{2}-1>0\right\} .
$$

Theorem 2.2 says that

$$
\iint_{C_{W}} \exp -\left[a_{1}^{2} x_{1}+a_{2}^{2} x_{2}-a_{1} a_{2}+\frac{1}{4 x_{1} x_{2}-1}\left(b_{1}^{2} x_{2}+b_{2}^{2} x_{1}+b_{1} b_{2}\right)\right] \frac{d x_{1} d x_{2}}{\sqrt{4 x_{1} x_{2}-1}}=\frac{\pi}{2} \frac{e^{-a_{1} b_{1}-a_{2} b_{2}}}{a_{1} a_{2}} .
$$

A warning: the extension of $M R I G_{n}$ to the case where some $b_{i}$ 's are negative leads to a non elementary elementary integral. The case $n=2$ is appropriate for explaining this fact: following the steps of the proof of Theorem 2.2 we arrive up to a multiplicative constant to the integral

$$
e^{a_{1} a_{2}} \int_{0}^{\infty} e^{-a_{1}^{2} x_{1}-\frac{a_{2}^{2}+b_{1}^{2}}{4 x_{1}}-a_{2}\left|b_{2}+\frac{b_{1}}{2 x_{1}}\right|} \frac{d x_{1}}{\sqrt{2 x_{1}}}
$$

that we cannot evaluate when $b_{1} b_{2}<0$.

\subsection{The complete graph for $n \geq 3$}

We consider the case where $w_{i j}=c$ for all $i \neq j$. Denote by $J_{n}$ the $n \times n$ matrix with all entries equal to 1. Therefore $W=c\left(J_{n}-I_{n}\right)$.

Proposition 3.1. If $W=c\left(J_{n}-I_{n}\right)$ then

$$
\begin{aligned}
\operatorname{det} M_{x} & =\left(c+2 x_{1}\right) \cdots\left(c+2 x_{n}\right)\left(1-\sum_{i=1}^{n} \frac{c}{c+2 x_{i}}\right) \\
C_{W} & =\left\{\left(x_{1}, \ldots, x_{n}\right) ; x_{1}, \ldots, x_{n} \geq 0, \sum_{i=1}^{n} \frac{c}{c+2 x_{i}}<1\right\} .
\end{aligned}
$$

Proof. Write $D=2 \operatorname{diag}\left(x_{1}, \ldots, x_{n}\right)+c I_{n}$. Therefore $M_{x}=D-c J_{n}=D^{1 / 2}\left(I_{n}-A\right) D^{1 / 2}$ where $A=$ $c D^{-1 / 2} J_{n} D^{-1 / 2}=v v^{\top}$ and

$$
v=\left(\frac{\sqrt{c}}{\sqrt{c+2 x_{1}}}, \ldots, \frac{\sqrt{c}}{\sqrt{c+2 x_{n}}}\right)^{\top} .
$$

The eigenvalues of $A$ are 0 with multiplicity $n-1$ and $v^{\top} v=\sum_{i=1}^{n} \frac{c}{c+2 x_{i}}$. This implies that the eigenvalues of $I_{n}-A$ are 1 with multiplicity $n-1$ and $1-\sum_{i=1}^{n} \frac{c}{c+2 x_{i}}$. This leads to (21). To prove (22), clearly the right-hand side contains $C_{W}$. Conversely if $x_{i}>0$ for all $i$ writing $M_{x}=D^{1 / 2}(I-A) D^{1 / 2}$ shows $x \in C_{W}$ if and only if $I-A$ is positive definite, i.e. $1-\sum_{i=1}^{n} \frac{c}{c+2 x_{i}}>0$. 


\subsection{The daisy}

We consider the case where

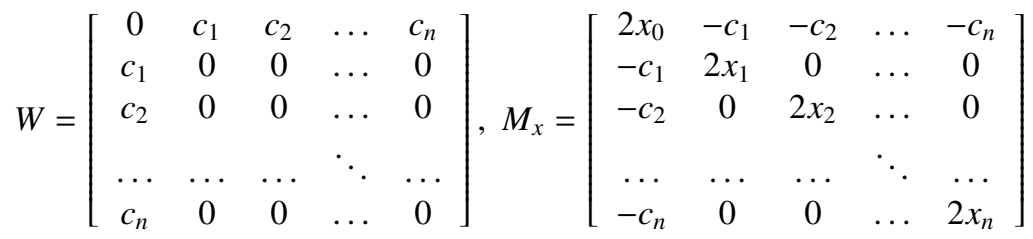

It is easy to see by induction that

$$
\begin{gathered}
\operatorname{det} M_{x}=2^{n} x_{1} \times \cdots \times x_{n}\left(2 x_{0}-\sum_{i=1}^{n} \frac{c_{i}^{2}}{2 x_{i}}\right), \\
C_{W}=\left\{\left(x_{0}, \ldots, x_{n}\right) ; x_{0}, \ldots, x_{n}>0,2 x_{0}-\sum_{i=1}^{n} \frac{c_{i}^{2}}{2 x_{i}}>0\right\} .
\end{gathered}
$$

It is elementary to write $M_{x}^{-1}$ explicitly. If we write for simplicity

$$
M(a, b, c)=\left[\begin{array}{ccccc}
a & c_{1} & c_{2} & \ldots & c_{n} \\
c_{1} & b_{1} & 0 & \ldots & 0 \\
c_{2} & 0 & b_{2} & \ldots & 0 \\
\ldots & \ldots & \ldots & \ddots & \ldots \\
c_{n} & 0 & 0 & \ldots & b_{n}
\end{array}\right]
$$

$B=b_{1} \times \cdots \times b_{n}, D=\operatorname{det} M(a, b, c)=B\left(a-\sum_{i=1}^{n} \frac{c_{i}^{2}}{b_{i}}\right)$ and $H=M(a, b, c)^{-1}=\left(h_{i j}\right)_{0 \leq i, j \leq n}$ then for $i \neq j$ and distinct from 0 we have

$$
h_{00}=\frac{B}{D}, h_{0 i}=-\frac{B}{D} \frac{c_{i}}{b_{i}}, h_{i i}=\frac{1}{b_{i}}\left(1+\frac{B}{D} \frac{c_{i}^{2}}{b_{i}}\right), h_{i j}=\frac{c_{i} c_{j}}{b_{i} b_{j}}\left(1+\frac{B}{D}\left(\frac{c_{i}^{2}}{b_{i}}+\frac{c_{j}^{2}}{b_{j}}\right)\right)
$$

For $n=2$ it gives $D=\operatorname{det} M_{x}=8 x_{0} x_{1} x_{2}-2 x_{2} c_{1}^{2}-2 x_{1} c_{2}^{2}$ and

$$
M_{x}^{-1}=\frac{1}{D}\left[\begin{array}{ccc}
4 x_{1} x_{2} & 2 c_{1} x_{2} & 2 c_{2} x_{1} \\
2 c_{1} x_{2} & 4 x_{0} x_{2}-c_{2}^{2} & c_{1} c_{2} \\
2 c_{2} x_{1} & c_{1} c_{2} & 4 x_{0} x_{1}-c_{1}^{2}
\end{array}\right]
$$

\subsection{The chain}

Define $A_{n+1}$ as the graph $\bullet-\bullet \cdot \stackrel{2}{\bullet}-\ldots-\stackrel{n}{\bullet}$ corresponding to the matrix

$$
W=\left[\begin{array}{ccccccc}
0 & c_{1} & 0 & 0 & \ldots & 0 & 0 \\
c_{1} & 0 & c_{2} & 0 & \cdots & 0 & 0 \\
0 & c_{2} & 0 & c_{3} & \ldots & 0 & 0 \\
0 & 0 & c_{3} & 0 & \cdots & 0 & 0 \\
\ldots & \ldots & \ldots & \ldots & \ddots & \ldots & \ldots \\
0 & 0 & 0 & 0 & \ldots & 0 & c_{n} \\
0 & 0 & 0 & 0 & \ldots & c_{n} & 0
\end{array}\right]
$$

where $c_{1}, \ldots, c_{n}>0$. Thus $M_{x}=-W+\operatorname{diag}\left(2 x_{0}, 2 x_{1}, \ldots, 2 x_{n}\right)$ is a Jacobi matrix. Without losing generality we may assume that $c_{1}=\ldots=c_{n}=1$ by the transformation

$$
\operatorname{diag}\left(\lambda_{0}, \lambda_{1}, \ldots, \lambda_{n}\right) M_{x} \operatorname{diag}\left(\lambda_{0}, \lambda_{1}, \ldots, \lambda_{n}\right)
$$


where

$$
\lambda_{0}=1, \lambda_{1}=\frac{1}{c_{1}}, \lambda_{2 p}=\frac{c_{1} c_{3} \ldots c_{2 p-1}}{c_{2} c_{4} \ldots c_{2 p}}, \lambda_{2 p+1}=\frac{c_{2} c_{4} \ldots c_{2 p}}{c_{1} c_{3} \ldots c_{2 p+1}}
$$

thus replacing $x_{i}$ by the affinity $x_{i} \lambda_{i}^{2}$. If $D_{0}=2 x_{0}$ and $D_{1}=4 x_{0} x_{1}-1$ then the determinant $D_{n}$ of the matrix

$$
M_{x}=\left[\begin{array}{ccccccc}
2 x_{0} & -1 & 0 & 0 & \ldots & 0 & 0 \\
-1 & 2 x_{1} & -1 & 0 & \cdots & 0 & 0 \\
0 & -1 & 2 x_{2} & -1 & \cdots & 0 & 0 \\
0 & 0 & -1 & 2 x_{3} & \cdots & 0 & 0 \\
\ldots & \ldots & \ldots & \ldots & \ddots & \ldots & \ldots \\
0 & 0 & 0 & 0 & \cdots & 2 x_{n-1} & -1 \\
0 & 0 & 0 & 0 & \cdots & -1 & 2 x_{n}
\end{array}\right]
$$

is computable by the induction formula $D_{n}=2 x_{n} D_{n-1}-D_{n-2}$. For instance for $n=3$ the set $C_{W}$ is described by the four inequalities

$$
x_{0}>0,4 x_{0} x_{1}-1>0,4 x_{0} x_{1} x_{2}-x_{0}-x_{2}>0,16 x_{0} x_{1} x_{2} x_{3}-4 x_{0} x_{3}-4 x_{2} x_{3}-4 x_{1} x_{2}+1>0 .
$$

Since the chain $A_{n+1}$ is also a tree, results of Section 5 below are applicable to this example.

\section{A study of the $M R I G_{n}$ distributions}

\section{1. $M R I G_{n}$ as a natural exponential family}

Writing $s_{i}=a_{i}^{2}$ in (7) we obtain

$$
\left(\frac{2}{\pi}\right)^{n / 2} \int_{C_{W}} e^{-\langle x, s\rangle-\frac{1}{2} b^{\top} M_{x}^{-1} b} \frac{d x}{\sqrt{\operatorname{det} M_{x}}}=\frac{1}{\sqrt{s_{1} \times \cdots \times s_{n}}} e^{-\left(b_{1} \sqrt{s_{1}}+\cdots+b_{n} \sqrt{s_{n}}\right)-\frac{1}{2} \sum_{i, j=1}^{n} w_{i j} \sqrt{s_{i} s_{j}}},
$$

which suggests that the natural exponential family (NEF) concentrated on $C_{W} \subset \mathbb{R}^{n}$ generated by the unbounded measure

$$
\mu(b, W)(d x)=e^{-\frac{1}{2} b^{\top} M_{x}^{-1} b} 1_{C_{W}}(x) \frac{d x}{\sqrt{\operatorname{det} M_{x}}}
$$

is interesting to study. For $n=1$ if $b_{1}>0$ this is nothing but a $R I G$ distribution mentioned in (3) and if $b_{1}=0$ it is a Gamma family with shape parameter $1 / 2$. For $n>1$ and $b=0$ this family is considered in Sabot, Tarrès and Zeng (2016). Given $W$ and $a \in(0,+\infty)^{n}, b \in[0,+\infty)^{n}$ we consider the probability on $[0,+\infty)^{n}$ defined by

$$
P(a ; b, W)(d x)=\left(\frac{2}{\pi}\right)^{n / 2}\left(\prod_{j=1}^{n} a_{j} e^{a_{j} b_{j}}\right) e^{-\frac{1}{2} a^{\top} M_{x} a-\frac{1}{2} b^{\top} M_{x}^{-1} b} \mathbf{1}_{C_{W}}(x) \frac{d x_{1} \times \cdots \times d x_{n}}{\sqrt{\operatorname{det} M_{x}}} .
$$

We say that $P(a ; b, W)$ is a $M R I G_{n}$ distribution. Theorem 2.2 proves that it is indeed a probability. From time to time we will use the notation $f(a ; b, W)(x)$ for the density of $P(a ; b, W)$. Note that $\left(X_{1}, \ldots, X_{n}\right) \sim$ $P(a ; b, 0)$ iff $X_{1}, \ldots, X_{n}$ are independent and $X_{k} \sim R I G\left(a_{k}, b_{k}\right), k \in\{1, \ldots, n\}$.

In this section, we show that if $X$ has a $M R I G_{n}$ distribution then the subvector $\left(X_{1}, \ldots, X_{k}\right)$ has a $M R I G_{k}$ distribution. We also show that up to a translation factor, the conditional distribution of $\left(X_{k+1}, \ldots, X_{n}\right)$ given $\left(X_{1}, \ldots, X_{k}\right)$ has a $M R I G_{n-k}$ distribution. Thus the class of $M R I G_{n}$ distributions has a remarkable property of stability by marginalization and conditioning. These facts have been independently observed by Sabot and Zeng (2019) in their Lemma 5, and also mentioned in Sabot and Zeng [15] quoting the arXiv versions of Sabot and Zeng [15] and of the present paper.

We begin with the calculation of the Laplace transform of $P(a ; b, W)$. Introducing the following function:

$$
G(a ; b, W)=\left(\frac{\pi}{2}\right)^{n / 2}\left(\prod_{j=1}^{n} \frac{e^{-a_{j} b_{j}}}{a_{j}}\right) e^{-\frac{1}{2} a^{\top} W a},
$$


we remark that $P(a ; b, W)(d x)$ can be written as

$$
P(a ; b, W)(d x)=\frac{1}{G(a ; b, W)} e^{-\left(x_{1} a_{1}^{2}+\cdots+x_{n} a_{n}^{2}\right)} \mu(b, W)(d x) .
$$

Under the form (26) we see that for fixed $b \in[0, \infty)^{n}$

$$
F_{b}=\left\{P(a ; b, W), a \in(0, \infty)^{n}\right\}
$$

is a natural exponential family, parameterized by $a$ and not by its natural parameter $\left(s_{1}, \ldots, s_{n}\right)=\left(a_{1}^{2}, \ldots, a_{n}^{2}\right)$, and generated by $\mu(b, W)$. From the fact that the mass of (26) is one, the Laplace transform of $\mu(b, W)$ is defined for $s \in(0, \infty)^{n}$ by

$$
L_{\mu(b, W)}(s)=G\left(\left(\sqrt{s_{1}}, \ldots, \sqrt{s_{n}}\right) ; b, W\right) .
$$

We deduce from this the form of the Laplace transform of $P(a ; b, W)$ itself.

Proposition 4.1. If $\left(X_{1}, \ldots, X_{n}\right) \sim P(a ; b, W)$ then

$$
\mathbb{E}\left(e^{-\left(s_{1} X_{1}+\cdots+s_{n} X_{n}\right)}\right)=e^{\langle a, b\rangle-\left\langle\sqrt{a^{2}+s}, b\right\rangle} e^{a^{\top} W a-\sqrt{a^{2}+s}{ }^{\top}} W \sqrt{a^{2}+s} \prod_{j=1}^{n} \frac{a_{j}}{\sqrt{a_{j}^{2}+s_{j}}},
$$

where we have written symbolically $\sqrt{a^{2}+s}=\left(\sqrt{a_{1}^{2}+s_{1}}, \ldots, \sqrt{a_{n}^{2}+s_{n}}\right)^{\top}$. In particular

$$
\begin{aligned}
\mathbb{E}\left(X_{i}\right)=m_{i} & =\frac{1}{2 a_{i}}\left(b_{i}+\sum_{j \neq i} w_{i j} a_{j}\right), \\
\operatorname{Var}\left(X_{i}\right) & =\frac{1}{4 a_{i}^{4}}+\frac{m_{i}}{2 a_{i}^{2}}, \\
\operatorname{Cov}\left(X_{i}, X_{j}\right) & =-\frac{w_{i j}}{4 a_{i} a_{j}}
\end{aligned}
$$

Comments. The one dimensional margins are the classical RIG distributions (3). More specifically the distribution of $X_{i}$ is

$$
R I G\left(a_{i}, b_{i}+\sum_{j=1}^{n} w_{i j} a_{j}\right)=R I G\left(a_{i}, 2 a_{i} m_{i}\right) .
$$

In other terms the two parameters of the distribution of $X_{i}$ 's are the $i$ components of the vectors $a$ and $b+W a$. Formula (28) expresses $m_{i}$ with a formula which is the successful change of variable (14) where the pair $(x, y)$ is replaced here by $(m, a)$. Observe also that the covariance of $\left(X_{i}, X_{j}\right)$ is never positive. It can be mentioned that, like for a Gaussian distribution, the parameters $(a, b, W)$ of the distribution $M R I G_{n}$ are determined if we know the distributions of all pairs $\left(X_{i}, X_{j}\right):$ the knowledge of the distribution of $X_{i}$ gives from (28) and (29) the knowledge of $m_{i}$ and $a_{i}$. The knowledge of the distribution of $\left(X_{i}, X_{j}\right)$ and of $a$ gives from (30) the knowledge of $w_{i j}$ and $W$, and then (28) gives the value of $b_{i}$. Estimation of the parameters can be designed from this remark. One more analogy with the Gaussian distributions is the fact that if $X \sim M R I G_{n}$ then $X_{i}$ and $X_{j}$ are independent if and only if they are uncorrelated: this can be read from the Laplace transform of $X$.

Proof of Proposition 4.1. Formula (27) comes immediately from

$$
\mathbb{E}\left(e^{-s_{1} X_{1}-\ldots-s_{n} X_{n}}\right)=\frac{\left.G\left(\sqrt{a_{1}^{2}+s_{1}}, \ldots, \sqrt{a_{n}^{2}+s_{n}}\right) ; b, W\right)}{G(a ; b, W)} .
$$

Equations (28) and (29) are consequence of the properties of the one dimensional $R I G$ given in (4). The simple formula (30) is obtained by $\left.\operatorname{Cov}\left(X_{i}, X_{j}\right)=\frac{\partial^{2}}{\partial s_{i} \partial s_{j}} \log G\left(\sqrt{a_{1}^{2}+s_{1}}, \ldots, \sqrt{a_{n}^{2}+s_{n}}\right) ; b, W\right)\left.\right|_{s=0}$. 
4.2. The marginals of the $M R I G_{n}$ distribution

For stating the next results we need the following notations:

- For vectors $\left(a_{1}, a_{2}, \ldots, a_{n}\right)^{\top}$ and $\left(b_{1}, b_{2}, \ldots, b_{n}\right)^{\top}$ we denote

$$
\tilde{a}_{k}=\left(a_{1}, \ldots, a_{k}\right)^{\top}, \tilde{b}_{k}=\left(b_{1}, \ldots, b_{k}\right)^{\top} .
$$

With this notation sometimes we write $P\left(\tilde{a}_{n} ; \tilde{b}_{n}, W\right)$ for $P(a ; b, W)$.

- If

$$
W_{n}=\left[\begin{array}{ccccc}
0 & w_{12} & w_{13} & \ldots & w_{1 n} \\
w_{12} & 0 & w_{23} & \ldots & w_{2 n} \\
\ldots & \ldots & \ldots & \ddots & \ldots \\
w_{1 n} & w_{2 n} & w_{3 n} & \ldots & 0
\end{array}\right]
$$

for $k \in\{2,3, \ldots, n\}$ we take $c_{k} \in \mathbb{R}^{k-1}$ defined as the $k$ th column of $W_{n}$ but restricted to be above the diagonal, namely $c_{k}=\left(w_{1 k}, w_{2 k}, \ldots, w_{k-1, k}\right)^{\top}$.

- If $k \in\{1,2,3, \ldots, n\}$ we write $W_{n}$ by blocks as follows

$$
W_{n}=\left[\begin{array}{cc}
W_{k} & W_{k, n-k} \\
W_{k, n-k}^{\top} & W_{n-k}^{\prime}
\end{array}\right] .
$$

In other terms $W_{k}=\left[w_{i j}\right]_{1 \leq i, j \leq k}, W_{n-k}^{\prime}=\left[w_{i j}\right]_{k+1 \leq i, j \leq n}$.

- The killing symbol $K$ from $\mathbb{R}^{n}$ to $\mathbb{R}^{n-1}$ is defined by

$$
K\left(x_{1}, \ldots, x_{n}\right)^{\top}=\left(x_{1}, \ldots, x_{n-1}\right)^{\top} .
$$

For instance $K \tilde{b}_{k}=\tilde{b}_{k-1}$. In general for $k<n$ we have

$$
K^{n-k}\left(x_{1}, \ldots, x_{n}\right)^{\top}=\left(x_{1}, \ldots, x_{k}\right)^{\top} .
$$

Proposition 4.2. If $\left(X_{1}, \ldots, X_{n}\right) \sim P\left(\tilde{a}_{n}, \tilde{b}_{n}, W\right)$ then $\left(X_{1}, \ldots, X_{k}\right) \sim P\left(\tilde{a}_{k}, B_{k}, W_{k}\right)$ where

$$
B_{k}=\tilde{b}_{k}+\sum_{j=k+1}^{n} a_{j} K^{j-k-1} c_{j}=\tilde{b}_{k}+W_{k, n-k}\left(a_{k+1}, \ldots, a_{n}\right)^{\top} .
$$

Proof. For $k=n-1$, this is claiming that $\left(X_{1}, \ldots, X_{n-1}\right) \sim P\left(\tilde{a}_{n-1}, \tilde{b}_{n-1}+a_{n} c_{n}, W_{n-1}\right)$. Such a formula is essentially formula (11) when replacing $n$ by $n+1$.

We now prove the result by induction on $n-k$. Suppose that $\left(X_{1}, \ldots, X_{k}\right) \sim P\left(\tilde{a}_{k}, B_{k}, W_{k}\right)$ is true. Then as for the passage from $n$ to $n-1$ we can claim that $\left(X_{1}, \ldots, X_{k-1}\right) \sim P\left(\tilde{a}_{k-1}, K B_{k}+a_{k} c_{k}, W_{k-1}\right)$. Now we have

$$
K B_{k}+a_{k} c_{k}=K \tilde{b}_{k}+a_{k} c_{k}+K \sum_{j=k+1}^{n} a_{j} K^{j-k-1} c_{j}=\tilde{b}_{k-1}+a_{k} c_{k}+\sum_{j=k+1}^{n} a_{j} K^{j-k} c_{j}=B_{k-1},
$$

and the induction is extended.

\section{Comments.}

- Proposition 4.2 could have been proved with the Laplace transform of Proposition 4.1, but is seems that after all induction is simpler. 
- A reformulation of Proposition 4.2 is the explicit form of the integral

$$
\int_{C_{W_{n-k}^{\prime}}} f_{\tilde{a}_{n}, \tilde{b}_{n}, W_{n}}\left(\tilde{x}_{k}, x_{k+1}, \ldots, x_{n}\right) d x_{k+1} \ldots d x_{n}=f_{\tilde{a}_{k}, B_{k}, W_{k}}\left(\tilde{x}_{k}\right),
$$

namely

$$
\begin{array}{r}
\left(\frac{2}{\pi}\right)^{n / 2}\left(\prod_{j=1}^{n} a_{j}\right) e^{\langle a, b\rangle-\frac{1}{2} a^{\top} W a} \int_{C_{W_{n-k}^{\prime}}} e^{-\left(x_{1} a_{1}^{2}+\cdots+x_{n} a_{n}^{2}\right)-\frac{1}{2} b^{\top} M_{x}^{-1} b} \mathbf{1}_{C_{W}}(x) \frac{d x_{k+1} \ldots d x_{n}}{\sqrt{\operatorname{det} M_{x}}} \\
=\left(\frac{2}{\pi}\right)^{k / 2}\left(\prod_{j=1}^{k} a_{j}\right) e^{\left\langle\tilde{a}_{k}, B_{k}\right\rangle} e^{-\frac{1}{2} \tilde{a}_{k}^{\top} M_{\tilde{x}_{k}} \tilde{a}_{k}-\frac{1}{2} B_{k}^{\top} M_{\tilde{x}_{k}}^{-1} B_{k}} \mathbf{1}_{C_{W_{k}}}\left(\tilde{x}_{k}\right) \frac{1}{\sqrt{\operatorname{det} M_{\tilde{x}_{k}}}} .
\end{array}
$$

- Inserting $b=0$ in Proposition 4.2 makes that $\left(X_{1}, \ldots, X_{n}\right)$ has an $S T Z_{n}$ distribution. If we also take $k=n-1$ we see that $B_{n-1}=a_{n} c$ where $c=\left(w_{i, n}\right)_{i=1}^{n-1}$. As a consequence, we see that any $M R I G_{n-1}$ distribution is a projection of some $S T Z_{n}$ distribution. This explains why Sabot, Tarrès and Zeng [17] indeed observe that one dimensional margins of an $S T Z_{n}$ distribution are $R I G$ ones.

\subsection{Conditional distributions under $M R I G_{n}$}

Let us begin by some general observations about exponential families on a product $E \times F$ of two Euclidean spaces generated by the distribution $\pi(d x) K(x, d y)$. Let $\Theta \subset E \times F$ be the interior of the set

$$
\left\{(t, s) ; L(t, s)=\int_{E \times F} e^{-\langle t, x\rangle-\langle s, y\rangle} \pi(d x) K(x, d y)<\infty\right\} .
$$

Let us assume that $\Theta$ is the product of two open subsets of $E$ and $F$ respectively:

$$
\Theta=\Theta_{E} \times \Theta_{F},
$$

let us fix $\left(t_{0}, s_{0}\right) \in \Theta$ and consider a random variable $(X, Y)$ valued in $E \times F$ with density

$$
\frac{1}{L\left(t_{0}, s_{0}\right)} e^{-\left\langle t_{0}, x\right\rangle-\left\langle s_{0}, y\right\rangle} \pi(d x) K(x, d y) .
$$

We are interested in the Laplace transform of the conditional distribution of $Y \mid X$. For computing this, consider the marginal density of $X$ with respect to $\pi$ :

$$
\frac{1}{L\left(t_{0}, s_{0}\right)} \int_{F} e^{-\left\langle t_{0}, x\right\rangle-\left\langle s_{0}, y\right\rangle} K(x, d y)=e^{-\left\langle t_{0}, x\right\rangle} \frac{g\left(s_{0} ; x\right)}{L\left(t_{0}, s_{0}\right)},
$$

where we have introduced the auxiliary function

$$
g\left(s_{0} ; x\right)=\int_{F} e^{-\left\langle s_{0}, y\right\rangle} K(x, d y)
$$

defined on $\Theta_{F} \times E$. As a consequence, the conditional distribution of $Y \mid X$ is $e^{-\left\langle s_{0}, y\right\rangle} K(X, d y) / g\left(s_{0} ; X\right)$ and its Laplace transform is for $s+s_{0} \in \Theta_{F}$ the ratio

$$
s \mapsto \frac{g\left(s+s_{0} ; X\right)}{g\left(s_{0} ; X\right)} .
$$

Suppose now that we are able to identify a density on $F$ having (32) as Laplace transform. In this case the problem of computation of the density of $Y \mid X$ will be solved.

This program will be applied to $E=\mathbb{R}^{k}, F=\mathbb{R}^{n-k}$, to a probability on $\mathbb{R}^{n}$ defined by its density

$$
f(x) \propto e^{-\frac{1}{2} b^{\top} M_{x}^{-1} b} \mathbf{1}_{C_{W}}(x) \frac{1}{\sqrt{\operatorname{det} M_{x}}}
$$


and finally to $t_{0}=\left(a_{1}^{2}, \ldots, a_{k}^{2}\right), s_{0}=\left(a_{k+1}^{2}, \ldots, a_{n}^{2}\right)$. We prove in Section 7 that $f$ is continuous on $\mathbb{R}^{n}$ when $b_{1}, \ldots, b_{n}$ is not zero and when the graph associated to $W$ is connected.

This condition (31) is fulfilled with $\Theta_{E}=(0, \infty)^{k}$ and $\Theta_{F}=(0, \infty)^{n-k}$. Of course $\tilde{X}_{k}=\left(X_{1}, \ldots, X_{k}\right)$ and $\left(X_{k+1}, \ldots, X_{n}\right)$ replace $X$ and $Y$. Also $s$ is now $\left(s_{k+1}, \ldots s_{n}\right)$ and $s+s_{0}$ is described by

$$
A_{k}(s)=\left(\sqrt{a_{k+1}^{2}+s_{k+1}}, \ldots, \sqrt{a_{n}^{2}+s_{n}}\right)^{\top} .
$$

The crucial function $g\left(s_{0} ; x\right)$ is now constructed from Proposition 4.2, where the marginal law of $\left(X_{1}, \ldots, X_{k}\right)$ is computed. We get

$$
g\left(a_{k+1}^{2}, \ldots, a_{n}^{2} ; \tilde{x}_{k}\right)=\frac{G(a ; b, W)}{G\left(\tilde{a}_{k} ; B_{k}, W_{k}\right)} \frac{e^{-\frac{1}{2} B_{k}^{\top} M_{\tilde{x}_{k}}^{-1} B_{k}}}{\sqrt{\operatorname{det}\left(M_{\tilde{x}_{k}}\right)}} \mathbf{1}_{C_{W_{k}}}\left(\tilde{x}_{k}\right),
$$

where the function $G(a ; b, W)$ has been introduced in (25). Remember that the right hand side of (33) depends of $a_{k+1}, \ldots, a_{n}$ also through $B_{k}=\tilde{b}_{k}+W_{k, n-k}\left(a_{k+1}, \ldots, a_{n}\right)^{\top}$. Let us adopt the notation

$$
B_{k}(s)=\tilde{b}_{k}+W_{k, n-k} A_{k}(s) .
$$

Here is now the Laplace transform of $\left(X_{k+1}, \ldots, X_{n}\right)$ given $\tilde{X}_{k}$

$$
\begin{gathered}
\mathbb{E}\left(e^{-s_{k+1} X_{k+1}-\ldots-s_{n} X_{n}} \mid \tilde{X}_{k}=\tilde{x}_{k}\right)=\frac{g\left(\left(a_{k+1}^{2}+s_{k+1}, \ldots, a_{n}^{2}+s_{n}\right) ; \tilde{x}_{k}\right)}{g\left(a_{k+1}^{2}, \ldots, a_{n}^{2} ; \tilde{x}_{k}\right)}=P Q R \\
P=\frac{G\left(\left(\tilde{a}_{k}, A_{k}(s)\right) ; b, W\right)}{G(a ; b, W)}, Q=\frac{G\left(\tilde{a}_{k} ; B_{k}, W_{k}\right)}{G\left(\tilde{a}_{k} ; B_{k}(s), W_{k}\right)}, R=e^{-\frac{1}{2} B_{k}(s)^{\top} M_{\tilde{X}_{k}}^{-1} B_{k}(s)+\frac{1}{2} B_{k}^{\top} M_{\tilde{x}_{k}}^{-1} B_{k}} .
\end{gathered}
$$

It is our intention to prove the existence of $\alpha=\left(\alpha_{k+1}, \ldots, \alpha_{n}\right)^{\top}, \beta=\left(\beta_{k+1}, \ldots, \beta_{n}\right)^{\top}, \gamma=\left(\gamma_{k+1}, \ldots, \gamma_{n}\right)^{\top}$ and of a matrix $\mathcal{W}$ such that

$$
P Q R=\frac{G\left(\sqrt{\alpha_{k+1}^{2}+s_{k+1}}, \ldots, \sqrt{\alpha_{n}^{2}+s_{n}} ; \beta, \mathcal{W}\right)}{G\left(\alpha_{k+1}, \ldots, \alpha_{n} ; \beta, \mathcal{W}\right)} e^{-\gamma_{k+1} s_{k+1}-\ldots-\gamma_{n} s_{n}}
$$

which is saying that the conditional distribution of $\left(X_{k+1}-\gamma_{k+1}, \ldots, X_{n}-\gamma_{n}\right)$ given $\tilde{X}_{k}$ is a $M R I G_{n-k}$ distribution. The next proposition gives the complete result:

Proposition 4.3. For $X \sim P(a ; b, W)$ consider $\alpha=\left(a_{k+1}, \ldots, a_{n}\right)^{\top}, \beta \in \mathbb{R}^{n-k}$ defined by

$$
\beta=\left(b_{k+1}, \ldots, b_{n}\right)^{\top}+W_{n-k, k} M_{\tilde{X}_{k}}^{-1} \tilde{b}_{k},
$$

the matrix $D=\operatorname{diag}\left(\gamma_{k+1}, \ldots, \gamma_{n}\right)$ defined as the diagonal part of the matrix $W_{n-k, k} M_{\tilde{X}_{k}}^{-1} W_{k, n-k}$ and

$$
\mathcal{W}=W_{n-k}^{\prime}+W_{n-k, k} M_{\tilde{X}_{k}}^{-1} W_{k, n-k}-D
$$

Then the conditional distribution of $\left(X_{k+1}-\gamma_{k+1}, \ldots, X_{n}-\gamma_{n}\right)$ given $\tilde{X}_{k}$ is $P(\alpha, \beta, \mathcal{W})$.

Proof. We have to analyze the dependency on $s$ of the three quantities $P, Q, R$ defined above by (35). However for simplification, we do not write the factors which do not depend on $s$. More specifically we introduce the following equivalence relation among non-zero functions $f$ or $g$ depending on $s$ and possibly on other parameters like $a, b, W$ by writing $f \equiv g$ if $f(s) / g(s)$ does not depend on $s$. For instance

$$
\begin{aligned}
P & \equiv \prod_{j=k+1}^{n}\left(a_{j}^{2}+s_{j}\right)^{-\frac{1}{2}} \times e^{-\left(b_{k+1} \sqrt{a_{k+1}^{2}+s_{k+1}}+\cdots+b_{n} \sqrt{\left.a_{n}^{2}+s_{n}\right)}\right.} e^{-\tilde{a}_{k}^{\top} W_{k, n-k} A_{k}(s)-\frac{1}{2} A_{k}(s)^{\top} W_{n-k}^{\prime} A_{k}(s)}, \\
Q & \equiv e^{\tilde{a}_{k}^{\top} W_{k, n-k} A_{k}(s)}, R \equiv e^{-\frac{1}{2} B_{k}(s)^{\top} M_{\hat{X}_{k}}^{-1} B_{k}(s)} \equiv e^{-A_{k}(s)^{\top} W_{n-k, k} M_{\bar{X}_{k}}^{-1} \tilde{b}_{k}} \times e^{-\frac{1}{2} A_{k}(s)^{\top} W_{n-k, k} M_{\dot{X}_{k}}^{-1} W_{k, n-k} A_{k}(s)} .
\end{aligned}
$$

A patient analysis of the product $P Q R$ as a function of $A_{k}(s)$ gives Proposition 4.3. 
4.4. A convolution property of the $M R I G_{n}$ laws

The following proposition is a generalization of the following additive convolution:

$$
R I G(a, b) * I G\left(a, b^{\prime}\right)=R I G\left(a, b+b^{\prime}\right)
$$

(see Barndorff-Nielsen and Koudou [3], Barndorff-Nielsen and Rydberg [4] and Barndorff-Nielsen, Blaesild and Seshadri [2]) with definitions in (2) and (3).

Proposition 4.4. Let $a_{i}, b_{i}, b_{i}^{\prime}>0$ for $i \in\{1, \ldots, n\}$. If $X=\left(X_{1}, \ldots, X_{n}\right)$ has the $M R I G_{n}$ distribution $P(a ; b, W)$, if $Y=\left(Y_{1}, \ldots, Y_{n}\right)$ such that $Y_{i} \sim I G\left(a_{i}, b_{i}^{\prime}\right)$ with independent components, and if $X$ and $Y$ are independent, then

$$
X+Y=\left(X_{1}+Y_{1}, \ldots, X_{n}+Y_{n}\right) \sim P\left(a ; b+b^{\prime}, W\right) .
$$

Proof. Compute the Laplace transform, using Proposition 4.1 and (2).

\subsection{Questions}

Here are some unsolved problems linked to $M R I G_{n}$ laws:

- If $X \sim P(a ; b, W)$ what is the distribution of $M_{X}^{-1}$ ? This random matrix is concentrated on a manifold of dimension $n$. This is a natural question since in one dimension if $X \sim R \operatorname{RG}(a, b)$ then the distribution of $1 / X$ is known and is $I G(b / 2,2 a)$. However the Laplace transform of $M_{X}^{-1}$, namely $L(s)=\mathbb{E}\left(e^{-\operatorname{tr}\left(s M_{X}^{-1}\right)}\right)$ defined when $s$ is a positive definite matrix of order $n$ is not known in general. If $b=0$ then $X$ has an $S T Z_{n}$ distribution and Theorem 2.2 shows that $L(s)$ is known for $s$ of rank one.

- Since in one dimension $I G$ and $R I G$ distributions are particular cases of the generalized inverse Gaussian laws, the natural extension of the $M R I G_{n}$ laws is to consider the probability densities on $\mathbb{R}^{n}$ proportional to

$$
e^{-\frac{1}{2} a^{\top} M_{x} a-\frac{1}{2} b^{\top} M_{x}^{-1} b}\left(\operatorname{det} M_{x}\right)^{q-1} \mathbf{1}_{\mathbf{C}_{\mathbf{W}}}(\mathbf{x})
$$

extending our familiar $M R I G_{n}$ integral from $1 / 2$ to an arbitrary real number $q$. But the corresponding integral extending Theorem 2.2 is untractable. However, in a particular case, namely if $b=0$ and if the graph $G$ associated to $W$ is a tree, Proposition 5.1 below computes the integral on $C_{W}$ of the function (36). A related distribution has been analyzed in Massam and Wesołowski [11] in connection with a multivariate version of the Matsumoto-Yor property (see e.g. Matsumoto and Yor [13], Letac and Wesołowski [10] and Massam and Wesołowski [12]).

- Probabilistic interpretations of the one dimensional laws $I G$ and $R I G$ are known, as hitting time and time of last visit of an interval $[a, \infty)$ by a drifted Brownian motion $t \mapsto m t+B(t)$ (in the respective cases $m>0$ and $m<0$ ). How to extend this to $M R I G_{n}$ laws? An answer to this problem is given in Sabot and Zeng (2017) but one may look for other interpretations.

\section{Another generalization of the Sabot-Tarrès-Zeng integral: the case of a tree}

In this section we consider another generalization of a specialization of the Sabot-Tarrès-Zeng integral (1): we assume that the graph $G$ associated to $W$ is a tree but we replace in (1) the power $-1 / 2$ of det $M_{x}$ by the real number $q-1>-1$. Furthermore in Proposition 5.2, we are able to drop the restriction $w_{i j} \geq 0$ that we have done all along the paper, because of the following proposition of linear algebra:

Proposition 5.1. Let $M=\left(m_{i j}\right)_{1 \leq i, j \leq n}$ be a symmetric matrix and let

$$
E=\left\{(i, j) ; 1 \leq i<j \leq n, m_{i j} \neq 0\right\} .
$$

Assume that $G$ is a graph with set of vertices $\{1, \ldots, n\}$ and with $E$ as set of edges. Then

(i) If $G$ is a tree or a forest, $\operatorname{det} M$ is a polynomial in $\left(m_{i i}\right)_{i=1}^{n}$ and in $\left(m_{i j}^{2}\right)_{(i, j) \in E}$; 
(ii) if $G$ is a tree or a forest, if $M$ is positive definite and if $\left(\epsilon_{i j}\right)_{1 \leq i, j \leq n}$ is a symmetric matrix such that $\epsilon_{i j}= \pm 1$ and $\epsilon_{i i}=1$ for all $i, j$ then the symmetric matrix $\left(\epsilon_{i j} m_{i j}\right)_{1 \leq i, j \leq n}$ is also positive definite;

(iii) if the graph has a cycle then $\operatorname{det} M$ is a sum of monomials such that at least one of them contains an odd power of some $m_{i j}$ with $i, j \in E$.

Comments. In general, changing the two off-diagonal entries $m_{i j}$ and $m_{j i}$ of a positive definite matrix $M$ into $-m_{i j}$ and $-m_{j i}$ creates a new symmetric matrix which can be not positive definite anymore. The proposition shows that this is not the case when the graph associated to $M$ is a tree. Part 3 shows that the fact that det $M$ is a polynomial in $\left(m_{i j}^{2}\right)_{I, J} \in E$ characterizes the fact that the graph is a tree or a forest.

Proof. We prove (i) by induction on $n$. The result is clear when $n=1$ and $n=2$. Suppose that it is true for $n$ and consider the case of a symmetric matrix $M_{1}$ of order $n+1$ such that its associated graph $G_{1}$ is a tree. Without loss of generality, we may assume that $n+1$ has only one neighbour in the tree and that this neighbor is $n$. This implies that $M_{1}$ has the form

$$
M_{1}=\left[\begin{array}{cc}
M & v \\
v^{\top} & m_{n+1, n+1}
\end{array}\right], \quad v^{\top}=\left(0, \ldots, 0, m_{n+1, n}\right),
$$

where the symmetric matrix $M$ of order $n$ is associated to the graph $G$ which is $G_{1}$ minus the vertex $n+1$. Since $n+1$ had $n$ as the only neighbour, $G$ is also a tree. Write also

$$
M=\left[\begin{array}{cc}
M_{-1} & v_{-1} \\
v_{-1}^{\top} & m_{n, n}
\end{array}\right],
$$

where $M_{-1}$ is symmetric of order $n-1$. Assume that $m_{n+1, n+1} \neq 0$ and denote $c=m_{n+1, n}^{2} / m_{n+1, n+1}$ and

$$
M^{\prime}=\left[\begin{array}{cc}
M_{-1} & v_{-1} \\
v_{-1}^{\top} & m_{n, n}-c
\end{array}\right] .
$$

Therefore we get that

$$
\operatorname{det} M_{1}=m_{n+1, n+1} \operatorname{det} M^{\prime} \text {. }
$$

Since $M^{\prime}$ is symmetric and since its associated graph is the tree $G$, the induction hypothesis implies that $\operatorname{det} M^{\prime}$ is a polynomial with respect to the squares of the $m_{i j}$ with $1 \leq i<j \leq n$ where $(i, j)$ is an edge of $G$. Also, det $M^{\prime}$ is an affine function of $c$. Since $c$ is a multiple of $m_{n+1, n}^{2}$, therefore the extension of the induction hypothesis to $n+1$ is done when $m_{n+1, n+1} \neq 0$. The extension to the case $m_{n+1, n+1}=0$ is done by continuity of the polynomial det $M_{1}$.

For showing (ii) we now apply (i) to the case where $M$ is positive definite and we assume without loss of generality that $G$ is a tree. We number its vertices $\{1, \ldots, n\}$ such that if $G_{k}$ is the graph associated to the restriction $M_{k}$ of $M$ to $\{1, \ldots, k\}^{2}$, then $G_{k}$ is a tree, a point which can be proved by induction. Denote $M_{k}(\epsilon)=\left(\epsilon_{i j} m_{i j}\right)_{1 \leq i, j \leq k}$. Since $G_{k}$ is a tree and since $M$ is positive definite, then $\operatorname{det}\left(M_{k}(\epsilon)\right)>0$. From the theorem of principal determinants, $M_{n}(\epsilon)$ is positive definite.

For showing (iii) we assume first that $G$ contains the cycle $1-2-\ldots-n-1$. We choose $m_{i j}=0$ if $|i-j| \neq 1 m_{12}=m_{21}=a$ and $m_{i, j}=1$ for the other edges of the cycle. With this choice the matrix $M$ is

$$
M_{n}=\left[\begin{array}{ccccccc}
0 & a & 0 & 0 & \ldots & 0 & 1 \\
a & 0 & 1 & 0 & \ldots & 0 & 0 \\
0 & 1 & 0 & 1 & \ldots & 0 & 0 \\
0 & 0 & 1 & 0 & \ldots & 0 & 0 \\
\ldots & \ldots & \ldots & \ldots & \ddots & \ldots & \ldots \\
0 & 0 & 0 & 0 & \ldots & 0 & 1 \\
1 & 0 & 0 & 0 & \ldots & 1 & 0
\end{array}\right] .
$$

Standard techniques show that $\operatorname{det} M_{n}=\operatorname{det} M_{n+4}$ for $n \geq 3$ and that

$$
\operatorname{det} M_{3}=\operatorname{det} M_{5}=2 a, \operatorname{det} M_{4}=(a-1)^{2}, \operatorname{det} M_{6}=-(a+1)^{2} .
$$


Therefore one of the monomials is $\pm 2 a$ : this odd power is the one which was announced and this ends the proof of Proposition 5.1.

For stating Proposition 5.2 we need to introduce the MacDonald function on $(0, \infty)$ :

$$
K_{q}(x)=\frac{1}{2} \int_{0}^{\infty} u^{q-1} e^{-\frac{1}{2} x\left(u+\frac{1}{u}\right)} d u
$$

It is useful to display a property of this integral

$$
2\left(\frac{b}{a}\right)^{q} K_{q}(2 a b)=\int_{0}^{\infty} v^{q-1} e^{-a^{2} v-\frac{b^{2}}{v}} d v .
$$

We denote by $s(i)$ the number of neighbours of $i$ in the tree, namely the size of $\left\{j: w_{i j}>0\right\}$.

Proposition 5.2. Let $W=\left(w_{i j}\right)_{1 \leq i, j \leq j}$ be a symmetric matrix with zero diagonal such that its associated graph $G$ is a tree. Let $M_{x}=2 \operatorname{diag}\left(x_{1}, \ldots, x_{n}\right)-W$ and let $C_{W}$ be the set of $x$ 's such that $M_{x}$ is positive definite. If $q>0$ then

$$
\int_{C_{W}} e^{-\frac{1}{2} a^{\top} M_{x} a}\left(\operatorname{det} M_{x}\right)^{q-1} d x=2^{q-1} \Gamma(q) e^{\frac{1}{2} a^{\top} W a} \prod_{i=1}^{n} a_{i}^{q(s(i)-2)} \prod_{i<j}\left|w_{i j}\right|^{q} K_{q}\left(a_{i} a_{j}\left|w_{i j}\right|\right) .
$$

\section{Comments.}

- For $y_{1}, \ldots, y_{n}>0$ another presentation of (38) is

$$
\int_{C_{W}} e^{-\langle x, y\rangle}\left(\operatorname{det} M_{x}\right)^{q-1} d x=2^{q-1} \Gamma(q) \prod_{i=1}^{n} y_{i}^{\frac{1}{2} q(s(i)-2)} \prod_{i<j} w_{i j}^{q} K_{q}\left(\sqrt{y_{i} y_{j}} w_{i j}\right) .
$$

- Of course, inserting $q=1 / 2$ gives back the Sabot -Tarrès -Zeng-integral in the case where $G$ is a tree. To check this we use Lemma 2.1 above which says

$$
K_{1 / 2}(x)=\sqrt{\frac{\pi}{2 x}} e^{-x}, \quad x>0 .
$$

For $q=3 / 2$ we use Watson [19] page 90 formula 12 for getting

$$
K_{3 / 2}(x)=\sqrt{\frac{\pi}{2 x}} e^{-x}\left(1+\frac{1}{x}\right), \quad x>0,
$$

and we obtain

$$
\int_{C_{W}} e^{-\frac{1}{2} a^{\top} M_{x} a} \sqrt{\operatorname{det} M_{x}} d x=\left(\frac{\pi}{2}\right)^{n / 2} \prod_{i=1}^{n} a_{i}^{-3} \prod_{i<j}\left(1+a_{i} a_{j} w_{i j}\right)
$$

- We give a proof of Proposition 5.1, while another proof could be extracted from Massam and Wesołowski (2004), where the authors consider the NEF generated by the unbounded measure

$$
1_{C_{W}}(x)\left(\operatorname{det} M_{x}\right)^{q-1} d x
$$

and independence properties of distributions from this NEF. Bobecka [5] has a multivariate generalization.

Proof. We proceed by induction on $n$. This is correct for $n=1$ since in this case $s(1)=0$ and since the empty product $\prod_{i<j}$ is one. Suppose that the formula (38) is true for $n$ and let us extend it to $n+1$. We use the same notation as in Section 2.2: we keep the notations $a, W$ and $M_{x}$ for the matrices of order $n$ as before 
and we consider the block matrices $M^{1}$ and $W^{1}$ defined by (8). We now use a different factorization of $M^{1}$ by writing

$$
M^{1}=\left[\begin{array}{cc}
I_{n} & -\frac{c}{2 x_{n+1}} \\
0 & 1
\end{array}\right]\left[\begin{array}{cc}
M_{x}-\frac{c c^{\top}}{2 x_{n+1}} & 0 \\
0 & 2 x_{n+1}
\end{array}\right]\left[\begin{array}{cc}
I_{n} & 0 \\
-\frac{c^{\top}}{2 x_{n+1}} & 1
\end{array}\right] .
$$

Since the graph $G^{1}$ which is associated to $W^{1}$ is a tree, without loss of generality we assume that the vertex $n+1$ has only one neighbour which is $n$. In other terms, we may assume that the vector $c$ of $\mathbb{R}^{n}$ has the form

$$
c=\left(0, \ldots, 0, w_{n, n+1}\right)^{\top} .
$$

This choice implies also that the graph $G$ associated to $W$ is still a tree. Formula (39) implies that $C_{W^{1}}$ is the set of $\left(x, x_{n+1}\right) \in \mathbb{R}^{n+1}$ such that $x_{n+1}>0$ and such that the diagonal $y$ of the matrix

$$
M_{y}=M_{x}-\frac{c c^{\top}}{2 x_{n+1}}=M_{x}-\left[\begin{array}{cc}
0 & 0 \\
0 & \frac{w_{n, n+1}^{2}}{2 x_{n+1}}
\end{array}\right] \text {, }
$$

namely $y=\left(x_{1}, \ldots, x_{n-1}, x_{n}-\frac{w_{n, n+1}^{2}}{4 x_{n+1}}\right)^{\top}$, belongs to $C_{W}$. The Jacobian of the transformation $\left(x, x_{n+1}\right) \mapsto$ $\left(y, x_{n+1}\right)$ is one. Therefore we can write

$$
\begin{aligned}
& \int_{C_{W^{1}}} e^{-\frac{1}{2} a^{\top} M_{x} a+a^{\top} c a_{n+1}-a_{n+1}^{2} x_{n+1}}\left(\operatorname{det} M^{1}\right)^{q-1} d x d x_{n+1} \\
= & e^{a^{\top} c a_{n+1}} \int_{C_{W^{1}}} e^{-\frac{1}{2} a^{\top}\left(M_{x}-\frac{c c^{\top}}{2 x_{n+1}}\right) a-\frac{\left(c^{\top} a\right)^{2}}{4 x_{n+1}}-a_{n+1}^{2} x_{n+1}} \operatorname{det}\left(M_{x}-\frac{c c^{\top}}{2 x_{n+1}}\right)^{q-1}\left(2 x_{n+1}\right)^{q-1} d x d x_{n+1} \\
= & \left(\int_{C_{W}} e^{-\frac{1}{2} a^{\top} M_{y} a}\left(\operatorname{det} M_{y}\right)^{q-1} d y\right) \times\left(e^{a^{\top} c a_{n+1}} \int_{0}^{\infty} e^{-\frac{\left(c^{\top} a\right)^{2}}{4 x_{n+1}}-a_{n+1}^{2} x_{n+1}}\left(2 x_{n+1}\right)^{q-1} d x_{n+1}\right) .
\end{aligned}
$$

The latter integral is expressed with (37) as

$$
e^{\left|w_{n, n+1}\right| a_{n} a_{n+1}} \frac{a_{n}^{q}}{a_{n+1}^{q}}\left|w_{n, n+1}\right|^{q} K_{q}\left(a_{n} a_{n+1}\left|w_{n, n+1}\right|\right),
$$

and the former one is (38), from the induction hypothesis. To conclude, observe that the number of neighbours of $n+1$ in $G^{1}$ is one, and that the number of neighbours of $n$ in $G^{1}$ is the number $s(n)$ of neighbours of $n$ in $G$ plus one.

\section{If $B \sim N\left(0, M_{x}\right)$ what is $\operatorname{Pr}\left(B_{1}>0, \ldots, B_{n}>0\right)$ ?}

Of course the exact solution of this question cannot be found. However for any $x \in C_{W}$ denote by

$$
f(x)=\operatorname{Pr}\left(B_{1}>0, \ldots, B_{n}>0\right) .
$$

Then formula (23) enables us to compute the Laplace transform of $f(x) \mathbf{1}_{C_{W}}(x)$. The trick is to observe that the first member of (23) involves the density $g_{x}(b)$ of $N\left(0, M_{x}\right)$ when the $b_{1}, \ldots, b_{n}$ are restricted to be $>0$. Of course $f(x)=\int_{\mathbb{R}_{+}^{n}} g_{x}(b) d b$, and $b \mapsto g_{x}(b) / f(x)$ is a probability density on $\mathbb{R}_{+}^{n}$.

One can even get a knowledge of the Laplace transform of $b \mapsto g_{x}(b)$. More specifically

Proposition 6.1. For $\theta=\left(\theta_{1}, \ldots, \theta_{n}\right) \in \mathbb{R}_{+}^{n}$ and $x \in C_{W}$, denote $\int_{\mathbb{R}_{+}^{n}} e^{-\langle\theta, b\rangle} g_{x}(b) d b=f(x, \theta)$. Then for $y=\left(y_{1}, \ldots, y_{n}\right) \in \mathbb{R}_{+}^{n}$ we have

$$
\int_{C_{W}} e^{-\langle x, y\rangle} f(x, \theta) d x=\frac{1}{2^{n} \sqrt{y_{1}\left(y_{1}+\theta_{1}\right)} \ldots \sqrt{y_{n}\left(y_{n}+\theta_{n}\right)}} e^{-\frac{1}{2} \sum_{i, j=1}^{n} w_{i j} \sqrt{y_{i} y_{j}}} .
$$

In particular 


$$
\int_{C_{W}} e^{-\langle x, y\rangle} f(x) d x=\frac{1}{2^{n} y_{1} \times \cdots \times y_{n}} e^{-\frac{1}{2} \sum_{i, j=1}^{n} w_{i j} \sqrt{y_{i} y_{j}}}
$$

Proof. Enough is to multiply both sides of (23) by $e^{-\langle\theta, b\rangle}$ and integrate with respect to $b$ on $\mathbb{R}_{+}^{n}$. Permuting the integrations on the left hand side leads to (40).

\section{Corollary 6.2.}

$$
f(x)=\frac{1}{(2 \pi)^{n / 2}} \int_{C_{W} \cap\{t \leq x\}} \frac{d t}{\sqrt{\left(x_{1}-t_{1}\right) \ldots\left(x_{n}-t_{n}\right)} \sqrt{\operatorname{det} M_{t}}} .
$$

Proof. Denote

$$
h(x)=\frac{1}{\pi^{n / 2}\left(x_{1} \times \cdots \times x_{n}\right)^{1 / 2}} \mathbf{1}_{\mathbb{R}_{+}^{n}}(x), g(x)=\frac{1}{(2 \pi)^{n / 2}} \frac{1}{\sqrt{\operatorname{det} M_{x}}} \mathbf{1}_{C_{W}}(x) .
$$

Consider the Laplace transforms $L_{f}(y), L_{g}(y), L_{h}(y)$ defined for $y_{1}, \ldots, y_{n}>0$. They are respectively given by (40) with $\theta=0$, by the Sabot-Tarrès-Zeng integral (1) and by

$$
\int_{\mathbb{R}_{+}^{n}} e^{-\langle x, y\rangle} h(x)=\frac{1}{\sqrt{y_{1} \times \cdots \times y_{n}}} .
$$

As a consequence $L_{f}=L_{g} L_{h}$ which implies that $f$ is the convolution product of $g$ and $h$ and proves (41).

Corollary 6.3. With the notation $D_{y}=\operatorname{diag}\left(y_{1}, \ldots, y_{n}\right)$ and $y=M_{x}^{-1} b$, where $b=(1, \ldots, 1)^{\top}$, we have

$$
f(x)=\frac{1}{(2 \pi)^{n / 2}} \sqrt{y_{1} \times \cdots \times y_{n}} \int_{(0, \infty)^{n}}\left(\frac{\operatorname{det}\left(D_{u+y}^{-1}+L_{W}\right)}{\left.\prod_{i=1}^{n}\left(u_{i}\left(u_{i}+y_{i}\right)\right)\right)}\right)^{1 / 2} d u_{1} \ldots d u_{n}
$$

with the Laplacian $L_{W}$ defined in (13).

Proof. In (41) we make the change of variable introduced in Lemma 2.4, namely $s=M_{t}^{-1} b$. We also observe that again from Lemma 2.4 it follows that $M_{x}-M_{t}=D_{b}\left(D_{y}^{-1}-D_{s}^{-1}\right)$ and that

$$
\prod_{i=1}^{n}\left(x_{i}-t_{i}\right)=\frac{1}{2^{n}} \operatorname{det}\left(M_{x}-M_{t}\right)=\frac{1}{2^{n}} \operatorname{det} D_{b}\left(D_{y}^{-1}-D_{s}^{-1}\right)=\frac{1}{2^{n}} \prod_{i=1}^{n} b_{i} \frac{s_{i}-y_{i}}{s_{i} y_{i}} .
$$

Using the fact that $b=(1, \ldots, 1)^{\top}$ we get

$$
f(x)=\frac{1}{(2 \pi)^{n / 2}} \int_{y_{1}}^{\infty} \ldots \int_{y_{n}}^{\infty}\left(\operatorname{det}\left(D_{s}^{-1}+L_{W}\right) \prod_{i=1}^{n} \frac{y_{i}}{s_{i}\left(s_{i}-y_{i}\right)}\right)^{1 / 2} d s_{1} \ldots d s_{n} .
$$

Hence the change of variables: $u_{i}=s_{i}-y_{i}, i \in\{1, \ldots, n\}$, yields (42).

\section{Comments.}

- Applying formula (41) even to the case $n=2$ is surprising, since the left hand side is explicitly known: recall that if

$$
\left(X_{1}, X_{2}\right) \sim N\left(0,\left[\begin{array}{cc}
1 & -\cos \alpha \\
-\cos \alpha & 1
\end{array}\right]\right) \Rightarrow \operatorname{Pr}\left(X_{1}>0, X_{2}>0\right)=\frac{\alpha}{2 \pi} .
$$

Therefore, if $M_{x}=\left[\begin{array}{cc}2 x_{1} & -w \\ -w & 2 x_{2}\end{array}\right]$ formula (41) gives the following double integral on the domain $D=\left\{\left(t_{1}, t_{2}\right), t_{1}<x_{1}, t_{2}<x_{2}, w<2 \sqrt{t_{1} t_{2}}\right\}:$

$$
\arccos \frac{w}{2 \sqrt{x_{1} x_{2}}}=\int_{D} \frac{d t_{1} d t_{2}}{\sqrt{\left(x_{1}-t_{1}\right)\left(x_{2}-t_{2}\right)\left(4 t_{1} t_{2}-w^{2}\right)}},
$$

an identity not so easy to check directly. 
- Some comments about tentative applications to Bayesian analysis of $M R I G_{n}$ are in order. Recall that a positive matrix $A=\rho I_{n}-C$ is called a $M$-matrix if $C=\left(c_{i j}\right)_{1 \leq i, j \leq n}$ is such that $c_{i j} \geq 0$ for all $i, j$. Of course with our usual notation and for $x \in C_{W}$ then $M_{x}$ is a $M$-matrix: we have just to define $c_{i j}=w_{i j}$ for $i \neq j, \rho=\max _{i} 2 x_{i}$ and $c_{i i}=\rho-2 x_{i}$ for seeing this fact. The $M$-matrices are widely used in statistics since for $X \sim N(0, \Sigma)$ then the density $g(x)$ of $X$ has the $M T P_{2}$ property, namely for all $x, y \in \mathbb{R}^{n}$

$$
g\left(\min \left(x_{1}, y_{1}\right) \ldots, \min \left(x_{n}, y_{n}\right)\right) g\left(\max \left(x_{1}, y_{1}\right) \ldots, \max \left(x_{n}, y_{n}\right)\right) \geq g(x) g(y)
$$

if and only if $\Sigma^{-1}$ is a $M$-matrix: we refer for instance to Karlin and Rinott (1983) Page 482 for this fact. In Theorem 3 of the same paper it is proved that for $X \sim N(0, \Sigma)$ and for all $i, j$ the covariance of $X_{i}, X_{j}$ conditioned by $\left\{X_{k} ; 1 \leq k \leq n, k \neq i, j\right\}$ is non negative if and only if $\Sigma^{-1}$ is a $M$-matrix. From the point of view of Bayesian analysis two types of Gaussian models come to mind

1. $\left\{N\left(0, M_{\theta}^{-1}\right) ; \theta \in C_{W}\right\}$. If $X \sim N\left(0, M_{\theta}^{-1}\right)$ its density is

$$
\frac{1}{(2 \pi)^{n / 2}} e^{-\frac{1}{2} x^{\top} M_{\theta} x} \sqrt{\operatorname{det} M_{\theta}}
$$

The densities have the $M T P_{2}$ property and the conditional covariances are all non negative. In order to use the $M R I G_{n}$ integral one is tempted to consider the a priori measure

$$
\pi(d \theta)=e^{-\frac{1}{2} b^{\top} M_{\theta}^{-1} b} 1_{C_{W}}(\theta) \frac{d \theta}{\operatorname{det} M_{\theta}},
$$

which is unfortunately unbounded since $x \mapsto \int_{C_{W}} N\left(0, M_{\theta}\right)(x) \pi(d \theta)$ is an unbounded density. From (23) and the last comment before the proof of Theorem 2.2, this density is proportional to $\prod_{i=1}^{n} e^{-a_{i}\left|x_{i}\right|} /\left|x_{i}\right|$.

2. $\left\{N\left(0, M_{\theta}\right) ; \theta \in C_{W}\right\}$. If $X \sim N\left(0, M_{\theta}\right)$ its density is

$$
\frac{1}{(2 \pi)^{n / 2}} e^{-\frac{1}{2} x^{\top} M_{\theta}^{-1} x} \frac{1}{\sqrt{\operatorname{det} M_{\theta}}}
$$

These densities have less attractive properties from the $M T P_{2}$ point of view. Nevertheless the a priori measure

$$
\pi(d \theta)=e^{-\left(a_{1}^{2} \theta_{1}+\cdots+a_{n}^{2} \theta_{n}\right)} 1_{C_{W}}(\theta) d \theta
$$

is bounded. However a major defect of this choice is the fact that $x \mapsto \int_{C_{W}} N\left(0, M_{\theta}^{-1}\right)(x) \pi(d \theta)$ is computable (by (23)) only if $x_{1}, \ldots, x_{n}$ are all non negative (again, see example $n=2$ in Section 3).

\section{Continuity of the density of the $M R I G_{n}$ laws}

Proposition 7.1. If $b_{1}, \ldots, b_{n} \geq 0$ with $b \neq 0$ and if the graph associated to $W$ is connected then the function

$$
f(x)=e^{-\frac{1}{2} b^{\top} M_{x}^{-1} b} \frac{1}{\sqrt{\operatorname{det} M_{x}}} 1_{C_{W}}(x)
$$

is continuous on $\mathbb{R}^{n}$.

Proof. The continuity of $f$ is clear outside of the boundary of $C_{W}$, namely outside of the set $\partial C_{W}$ of $x \in \mathbb{R}^{n}$ such that $M_{x}$ is positive semidefinite with det $M_{x}=0$. In the sequel we fix $x \in \partial C_{W}$ and we prove the continuity of $f$ at this point $x$.

FIRST STEP. We show that if $t=\left(t_{1}, \ldots, t_{n}\right)^{\top} \in \mathbb{R}^{n}$ is such that $M_{x} t=0$ and if $t_{i_{0}}>0$ for some $i_{0}$, then $t_{i}>0$ for all $i=1, \ldots, n$. To see this, we use the notation $t_{i}^{+}=\max \left(0, t_{i}\right), t_{i}^{-}=t_{i}^{+}-t_{i}$ and

$$
t^{+}=\left(t_{1}^{+}, \ldots, t_{n}^{+}\right)^{\top}, t^{-}=t^{+}-t .
$$


Since $0=M_{x} t=M_{x} t^{+}-M_{x} t^{-}$we multiply by $\left(t^{+}\right)^{\top}$ on the left for getting $\left(t^{+}\right)^{\top} M_{x} t^{+}=\left(t^{+}\right)^{\top} M_{x} t^{-}$. Since $t_{i}^{+} t_{i}^{-}=0$ we have that $\left(t^{+}\right)^{\top} M_{x} t^{-} \leq 0$. Since $M_{x}$ is positive semidefinite we have that $\left(t^{+}\right)^{\top} M_{x} t^{+}=0$ and therefore $M_{x} t^{+}=0$. Without loss of generality, assume that $t^{+}=\left(t_{1}, \ldots, t_{k}, 0, \ldots, 0\right)$ with $t_{1}, \ldots, t_{k}>0$. Let us show that $k=n$. Since $t_{i_{0}}>0$ we have $k>0$. Suppose that $k<n$. We now split $M_{x}$ in blocks

$$
M_{x}=\left[\begin{array}{cc}
A & B \\
B^{\top} & C
\end{array}\right],
$$

where $A$ is a $(k, k)$ matrix. Clearly since $M_{x} t^{+}=0$ we get $B^{\top}\left(t_{1}, \ldots, t_{k}\right)^{\top}=0$. Since it holds for all $t_{j}>0$ for $j \in\{1, \ldots, k\}$ this implies that $B=0$. This contradicts the fact that $G$ is connected, and finally $k=n$.

Second STEP. We show that no principal minor of $M_{x}$ of order $n-1$ can be zero. Suppose for instance that the cofactor $C_{i_{0}}(x)$ of $2 x_{i_{0}}$ is zero. This implies that there exists a non-zero $t \in \mathbb{R}^{n}$ such that $M_{x} t=0$ and $t_{i_{0}}=0$. From the first step, this is impossible.

THIRD STEP. Consider a sequence $\left(x_{k}\right)_{k=1}^{\infty}$ in $C_{W}$ converging to $x$ and let us show that $f\left(x_{k}\right)$ converges to zero. This is equivalent to show that

$$
E_{k}=b^{\top} M_{x_{k}}^{-1} b+\log \operatorname{det} M_{x_{k}} \rightarrow \infty .
$$

Recall that we have assumed that there exists $i_{0}$ such that $b_{i_{0}}>0$. Recall also that all coefficients of $M_{x_{k}}^{-1}$ are non-negative. As a consequence

$$
E_{k} \geq b_{i_{0}}^{2} C_{i_{0}}\left(x_{k}\right) \frac{1}{\operatorname{det} M_{x_{k}}}+\log \operatorname{det} M_{x_{k}} .
$$

As polynomials in $x_{k}$ we have that $C_{i_{0}}\left(x_{k}\right)$ converges to $C_{i_{0}}(x)$ and $\operatorname{det} M_{x_{k}}$ converges to $\operatorname{det} M_{x}$. Since $C_{i_{0}}\left(x_{k}\right)>0$ from the second step and since $\operatorname{det} M_{x}=0$, we have shown that $E_{k}$ tends to infinity and the proof is done.

\section{Acknowlegments}

We thank Christophe Sabot for having introduced the first author to these questions and for many useful comments. This research was partially supported for the second author by the project 2016/21/B/ST1/00005 of the National Science Center, Poland. The first author is grateful for the hospitality of the Faculty of Mathematics and Information Science of the Warsaw University of Technology.

[1] R.B. Bapat, Graphs and Matrices, Springer Universitext, Springer London Dordrecht Heidelberg NewYork (2010) .

[2] O.E. Barndorff-Nielsen, P. Blaesild, V. Seshadri, Multivariate distributions with generalized inverse Gaussian marginals and associated Poisson mixtures, Can. J. Statist. 20 (1992), 109-120.

[3] O.E. Barndorff-Nielsen, A.E. Koudou, Trees with random conductance and the (reciprocal) inverse Gaussian distribution, Adv. Appl. Probab. 30 (1998) 409-424.

[4] O.E. Barndorff-Nielsen, T.H. Rysberg, Exact distributional results for random resistance trees, Scand. J. Statist. 27 (2000) 129141.

[5] K. Bobecka, The Matsumoto-Yor property on trees for matrix variates of different dimensions, J. Multivar. Anal. 141 (2015) 22-44.

[6] G. Boole, Théorème général concernant l'intégration définie, J. Math. Pures Appl. (1), 13 (1848) 111-112.

[7] M. Disertori, T. Spencer, M.R. Zinbauer, Quasi Diffusion in a 3D Supersymmetry Hyperbolic Sigma Model, Comm. Math. Phys. 300 (2010) 435-486.

[8] M. Disertori, E.F. Merkl, S.W.W. Rolles, A supersymmetric approach to martingales related to the vertex-reinforced jump process, arXiv:1511.07157, ALEA Lat. J. Probab. Math. Stat. (2017) 529-555.

[9] S. Karlin, Y. Rinott, $M$-matrices as covariance matrices of multinomial distributions, Linear Algebra Appl. 52/53 (1983) 419-438.

[10] G. Letac, J. Wesołowski, An independence property for the product of GIG and gamma laws, Ann. Probab. 28 (2000) 1371-1383.

[11] H. Massam, J. Wesołowski, The Matsumoto-Yor property on trees, Bernoulli 10 (2004) 685-700.

[12] H. Massam, J. Wesołowski, The Matsumoto-Yor property and the structure of Wishart distributions, J. Multivariate Anal. 97 (2006) 103-123.

[13] H. Matsumoto, M. Yor, An analogue of Pitman's $2 M-X$ theorem for exponential Wiener functionals. Part II: The role of the generalized inverse Gaussian laws, Nagoya Math. J. 162 (2001) 65-68.

[14] C. Sabot, P. Tarrès, Edge-reinforced random walk, vertex-reinforced jump process and the supersymmetric sigma model, J. Eur. Math. Soc. 17 (2015) 2353-2378. 
[15] C. Sabot, X. Zeng, Hitting times of interacting drifted Brownian motions and the vertex reinforced jump process, arXiv: 1704.05394 (2017)

[16] C. Sabot, X. Zeng, A random Schrödinger operator associated with the Vertex Reinforced Jump Process on infinite graphs, J. Amer. Math. Soc. 32 (2019) 311-349.

[17] C. Sabot, P. Tarrès, X. Zeng, The vertex reinforced jump process and a random Schrodinger operator on finite graphs, Ann. Probab. 45 (2017) 3967-3986.

[18] V. Seshadri, The Inverse Gaussian distribution, Clarendon Press, Oxford (1993).

[19] G.N. Watson, A Treatise on the Theory of Bessel Functions, Cambridge University Press (1966). 